\title{
Transient Analysis of Idle Time in VANETs Using Markov-Reward Models
}

\author{
Isabel V. Martin-Faus ${ }^{(1)}$, Luis Urquiza-Aguiar ${ }^{(1)}$, Mónica Aguilar Igartua, and Isabelle Guérin-Lassous
}

\begin{abstract}
The development of analytical models to analyze the behavior of vehicular ad hoc networks (VANETs) is a challenging aim. Adaptive methods are suitable for many algorithms (e.g., choice of forwarding paths, dynamic resource allocation, channel control congestion) and services (e.g., provision of multimedia services, message dissemination). These adaptive algorithms help the network to maintain a desired performance level. However, this is a difficult goal to achieve, especially in VANETs due to fast position changes of the VANET nodes. Adaptive decisions should be taken according to the current conditions of the VANET. Therefore, evaluation of transient measures is required for the characterization of VANETs. In the literature, different works address the characterization and measurement of the idle (or busy) time to be used in different proposals to attain a more efficient usage of wireless network. This paper focuses on the idle time of the link between two VANET nodes, which we denote as $T_{i d l e}$. Specifically, we have developed an analytical model based on a straightforward Markov reward chain to obtain transient measurements of $T_{i d l e}$. Numerical results from the analytical model fit well with simulation results.
\end{abstract}

Index Terms-Analytical model, channel idle time, vehicular ad hoc networks (VANETs), Markov reward chain (MRC), Markovian model, performability.

\section{INTRODUCTION}

$\mathbf{T}$ HE development of analytical models to analyze the behavior of vehicular ad hoc networks (VANETs) is a challenging goal which raises an increasing interest in the research community. Models are designed to understand and evaluate the VANET performance, to efficiently provision the network and/or to propose adapted solutions to VANETs. In these networks, adapted solutions are often adaptive solutions.

Manuscript received March 30, 2016; revised February 27, 2017 and July 19, 2017; accepted October 3, 2017. Date of publication February 12, 2018; date of current version April 16, 2018. This work was supported in part by the Spanish Government through projects TEC2014-54335-C4-1-R "INcident monitoRing In Smart COmmunities. QoS and Privacy" (INRISCO), in part by TEC2015-70197-R "A Software Architecture for rate-control over integrated satellite-terrestrial networks" (ARPASAT), and in part by the Fonds Recherche of ENS Lyon. The work of L. Urquiza-Aguiar was supported by SENESCYT (Ecuador) with the sponsorship of Escuela Politécnica Nacional (EPN). The review of this paper was coordinated by Prof. Y. P. Fallah. (Corresponding author: Isabel V. Martin-Faus.)

I. V. Martin-Faus, L. Urquiza-Aguiar, and M. A. Igartua are with the Department of Network Engineering, Universitat Politècnica de Catalunya (UPC), Barcelona 08034, Spain (e-mail: isabel.martin@entel.upc.edu; luis.urquiza@ entel.upc.edu; monica.aguilar@entel.upc.edu).

I. Guérin-Lassous is with the University of Lyon, UCB Lyon 1, CNRS, ENS de Lyon, INRIA, LIP UMR 5668, Lyon 69364, France (e-mail: isabelle. guerin-lassous@ens-lyon.fr).

Color versions of one or more of the figures in this paper are available online at http://ieeexplore.ieee.org.

Digital Object Identifier 10.1109/TVT.2017.2766449
Adaptability must help to ensure a performance level whatever the network evolution. Adaptive methods are suitable for many specific issues, like, for example, the choice of forwarding paths, dynamic resource allocation, congestion control, provision of multimedia services, message dissemination control and improvement of medium reuse. Nevertheless, adaptability is a hard task because of the fast changes and unpredictable nature of a VANET. Adaptive decisions should be taken according to the current conditions of the VANET. Thus, analytical models that provide transient measure evaluations in VANETs are necessary.

A measurement that is strongly related to the communication channel state of a wireless network is the idle time (or its opposite busy time) on the channel, i.e., the time during which the shared wireless medium is free and available to be used by the nodes.

Idle (or busy) time measurements are a key step in wireless networks that can be used for different problems. For example, they can be useful to determine the remaining resources like, for instance, the available bandwidth in order to get an indication on the possibility to send (or not) more traffic into the network. This knowledge is useful in any wireless network and is required in cognitive radio networks. They can also be used to adjust different parameters in VANET nodes, such as power or safety messages generation rate.

Due to the dynamic feature and the use of the radio medium in VANETs, the bandwidth of the network links can greatly vary with time. Estimating how the available bandwidth evolves with time and on transient periods is a knowledge that can be used by the network to adapt its solutions to the remaining bandwidth. Available bandwidth is strongly related to idle periods, and, as far as we know, no solution has been proposed to estimate idle time in transient periods.

In the present work, we focus on the calculation of the total idle time over a measurement interval on a link composed by two nodes of a VANET. We assume that the VANET uses the IEEE 802.11p wireless technology, where the wireless medium is shared with a Carrier Sense Multiple Access/Collision Avoidance (CSMA/CA) policy. The total idle time of the link is the time during which the communication medium for both sender and receiver is free. Henceforth, we denote the total idle time as $T_{i d l e}$. To estimate $T_{i d l e}$, it is needed to consider the simultaneous silent intervals of the two nodes that form the communication link of a VANET. Due to the high changing conditions of a VANET, our aim is to obtain transient measures of $T_{i d l e}$. 
Specifically in this paper, we propose an analytical model to measure $T_{\text {idle }}$. To attain this goal, we have investigated the applicability of well-known performability techniques [1], [2]. In particular, we have considered Markov reward chains (MRCs) and the randomization method [3], [4] to evaluate the proposed model. These methodologies were employed in the communication research area, e.g., to evaluate connection admission control algorithms [5] or to evaluate adaptive-rate video-streaming services [6]. These tools allow us to develop a simple approach for the modeling. We obtain an MRC which needs only mean values from our system. All the parameters of our model have a clear relation to the link behavior in the VANET. Moreover, our modeling method allows us to consider the initial state of the link and its changes throughout time including the relative distance between the nodes of the link. Our model is compared with simulations. The obtained results show the model is accurate. The proposed model is thus a useful tool that can quickly estimate the idle time on any link of a VANET. The solution can easily be embedded in the nodes in order to let each node to compute its available bandwidth with its neighbors.

The rest of the paper is organized as follows. In Section II, previous works are pointed out. Section III describes the VANET scenario evaluated in this paper. The proposed analytical model is presented in Section IV. Numerical results from the VANET model are compared with simulations in Section V to verify its accuracy. Finally, conclusions and future work are drawn in Section VII.

\section{RELATED WORK}

Idle and busy times are important parameters, in networks based on a CSMA/CA approach, that impact the network bandwidth and the node throughput. Several solutions use the idle/busy time to estimate network performance. For instance, in [7], the authors propose a model to estimate the throughput of an individual node in an ad hoc network. Based on the characterization of a node using an ON-OFF process [8], they characterize the behavior of each node taking into account the backoff and the packet loss probabilities. An exhaustive classification of packet losses due to collisions is presented. As part of the model, they compute the fraction of busy time of a node.

Different works on available bandwidth estimation also calculate the idle time as part of their proposals [9], [10]. Most of them compute the available bandwidth as the maximum capacity weighted by the fraction of idle time over a measurement interval. Those approaches are based on an accurate modeling of the neighborhood, including influential factors such as collisions due to the different flow interferences and backoff time (in one or more of its contention phases). Since the measurement of the idle time is done separately at each node (sender and receiver) of a link, these approaches also apply different methods to capture the overlap between idle periods of both sender and receiver. These solutions are designed for relatively static networks and are not initially thought for VANETs. The ABE+ proposal in [11] is an adaptation of the solution described in [9] that is modified for VANET scenarios. In [9] the available bandwidth on the different links is estimated to be used in a multimetric routing protocol in order to take proper packet forwarding decisions. ABE+ considers special features of VANETs, especially the high speed of the nodes. In particular, a new function to estimate the packet collision probability is obtained. That function is derived from off-line statistical analysis of a data collection experimentally obtained from simulations in VANET urban scenarios. If $\mathrm{ABE}+$ improves the routing performance, the available bandwidth estimation, and thus the idle period estimation is rather a long term evaluation that does not express the idle period evolution during the transient periods encountered in VANETs.

In [12], the authors are also interested in the link performance in VANETs. They evaluate the communication performance on a specific link consisting of two vehicles. The evaluation is carried out experimentally with an obstacle vehicle that can be located between the two vehicles under study. The experiments bring very interesting results on the link performance in terms of received signal strength indicator (RSSI) and packet delivery ratio. Complementarily, in our proposal we analyze the idle periods and we propose a model to estimate those idle periods.

Estimating the idle period duration is also used in cognitive radio networks where coexistent networks reuse resources not only in the spatial domain (e.g., dynamic spectrum), but also in the temporal domain (and by exploiting idle periods) [13], [14]. The idle time and busy time distributions of the primary network are important parameters for cognitive radio networks. In these studies, the primary network is a wireless local area network in which at most one node can transmit at a given time, whereas, in our scenario, several nodes, far enough, may transmit in parallel. In [15], the authors study the idle time distribution in multihop wireless networks, but the targeted ad hoc scenarios are static and the study is not focused on transient measurements.

Idle and busy times are also used in several papers where the authors propose congestion control schemes by adjusting different parameters in VANET nodes, such as power or messages generation rate. The authors of [16] offer the possibility to use the channel busy ratio (in place of the aggregate offered load) as feedback measurement to design an adaptive message generation rate method to control the channel congestion. Similarly, the channel busy ratio is a key parameter in [17] that seeks to maximize the information dissemination rate as a function of the message generation rate and the transmission power of the individual VANET nodes. These two papers use the busy periods but do not propose models to analyze idle/busy times. In [18], the authors propose a model for VANETs in order to study the impact of transmission rate and transmission range on the VANET performance. This model is based on busy (idle) period estimation. To this end, the authors need to compute the hidden node collision probability. In our approach, we do not integrate as accurately as in [18] the impact of collisions because we think that this approach is complex for evaluating transient parameters.

Some papers propose models for VANETs in order to analyze the performance of the network or of some services that can be offered with these networks. For instance, the authors of [19] analyze the periodic broadcast that takes place in VANETs. The aim of this work is to calculate the performance of the periodic broadcast in terms of packet collision probability and 
average packet delay. They propose a Markovian model based on [20], which they modify to characterize the periodical broadcast under unsaturated conditions with deterministic message arrival. Also, they take into account the freezing of the backoff counter due to busy periods. In [21], the authors extend their previous work [20] by considering the hidden terminal problem and the station mobility in a highway scenario to determine the broadcast operation performance. They place vehicles on a onedimensional road according to a Poisson point process with a given density (in vehicles/meter). A constant relative velocity of vehicles is assumed. Also, they design a channel model for VANETs focusing on safety applications. The proposed analytical model includes the impact of several parameters on the performance like priority access, message arrival interval, hidden terminal problem, fading transmission channel and highly mobile vehicles. Later, in [22], the modeling of the vehicles' movement is improved by considering two lanes instead of one, and the probability of busy channel is obtained from the point of view of a particular node. The works in [23] and [24] consider a heterogeneous distribution of vehicular traffic. The former considers an urban scenario and the latter a highway. Specially, the mobility traffic model proposed in [24], which is based on a stochastic model, could be easily included in our modelling method. To simplify our proposal, we have started with a homogeneous mobility model and we let the consideration of heterogeneous mobility model for future work.

Nonetheless, though these related works use busy times to estimate performance of broadcast traffic, none of them analyze a particular link between a pair of nodes, as we do in this paper. Besides, these models are complex when evaluating transient measurements. Our proposal is simple enough to evaluate transient measures of the idle time on links formed by VANET nodes.

\section{THE VANET SCENARIO UNDER EVALUATION}

\section{A. The Scenario}

We assume that vehicles communicate with the IEEE $802.11 \mathrm{p}$ technology and that the Request To Send/Clear To Send (RTS/CTS) mechanism is not activated in the medium access control mechanism. In our study, to simplify the analysis, we assume that all nodes have the same transmission range, which is denoted as $\boldsymbol{r} \boldsymbol{t} \boldsymbol{x}$.

Our interest is focused on the estimation, during an observation interval $\boldsymbol{T}$, of the total idle time over a Link A-B, which we denote as $T_{\text {idle_AB }}$. We consider that the set of nodes located within the carrier sense range of a node is the neighborhood (or Ngh) of this node. We denote as Ngh-A (resp. Ngh-B) the A's (resp. B's) neighborhood. In order to know when the medium for Link A-B is idle, we need to identify any transmitting neighbor of node A or node B, because the contention mechanism used in IEEE $802.11 \mathrm{p}$ allows any node to start a packet transmission when the medium is idle, i.e., no neighbor node is transmitting. Note that we only consider, in this study, a perfect carrier sensing mechanism and that only $802.11 \mathrm{p}$ signals are transmitted on the network. The impact of imperfect carrier sensing on the idle time evaluation is left as a future work. The carrier sense range
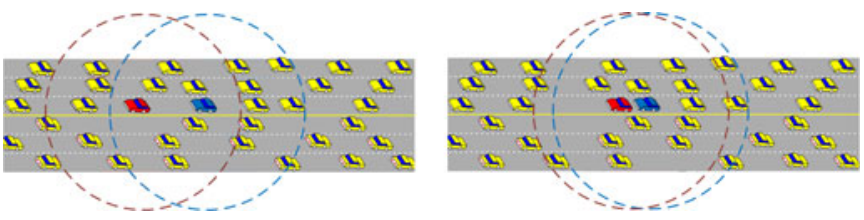

Fig. 1. Evaluated VANET scenario.

is assumed to be the same for all the nodes and it is denoted as $\boldsymbol{r} \boldsymbol{s} \boldsymbol{n}$. Finally, it will not be necessary to identify the receiver or to differentiate if the packet transmission succeeded or failed since we just want to measure if the medium is busy or idle.

Fig. 1 shows a generic scheme of a highway scenario where Link A-B formed between the red vehicle (node $\mathrm{A}$ ) and the blue vehicle (node B) is under analysis. The circles denote the limit of their respective carrier sense range. Differences between both images in Fig. 1 show the topology changes throughout time due to the relative movement of the vehicles. The distance between the two vehicles can change with time. We consider that the maximum distance between the two vehicles is such that both vehicles are still in transmission range of each other, just before link breakage.

In addition to the configuration values of the VANET, to evaluate transient measures, we need to identify which is the initial state of the system. Some of the variables whose value could change throughout time in the VANET are:

- Rate of packets that the node wants to transmit on the channel.

- Duration of the channel use each time the node accesses the channel.

- Number of neighbors sensed by the node.

- Speed of the nodes.

- Distance between the nodes.

In a real VANET, we could take advantage of the fact that VANET nodes periodically broadcast messages [25], in which these parameters could be included. Thus, each node can periodically gather updated information from its neighbor nodes and send them its own information. Note that two neighbors generally are the center of different Ngh since each node covers a different zone within its carrier sense range. Consequently, two neighbors may have different neighbors and therefore gather different information of the list mentioned above. For instance, the idle period that a node could locally perceive on its $\mathrm{Ngh}$ can be different when measured in nodes A and B of Link A-B. To calculate $T_{i d l e \_A B}$ we need to know when these idle periods simultaneously take place in both nodes, A and B.

\section{B. Geographical Location and Mobility of the Nodes}

We assume a knowledge on the density and velocity pattern of the vehicles. In particular, regarding the geographical location of the nodes within an Ngh, we approximate the Ngh with rows and columns. Examples of different distributions are shown in Fig. 2. We remind that the dotted circles indicate the carrier sense range which corresponds to the Ngh of each node. The way the neighbors are distributed in each row and column defines different types of models. We have called these models as 


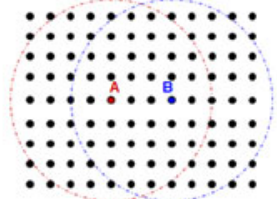

(a)
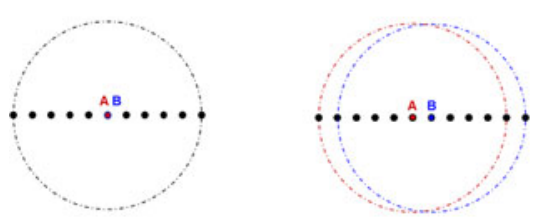

(c)
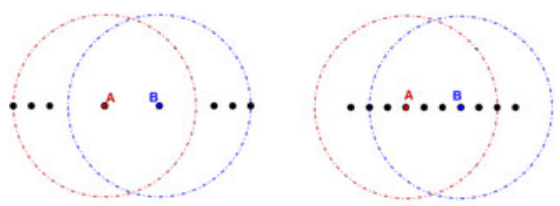

(d)

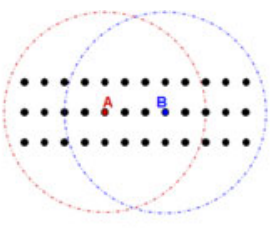

(b)
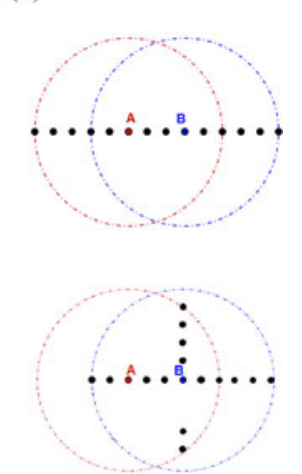

(1)

Fig. 2. Geographical distributions of nodes. (a) Homogeneous approximation. (b) Semi-homogeneous approximation for three lanes. (c) Semi-homogeneous approximation for one lane. Evolution of the relative distance from minimum to maximum distance between nodes A and B. (d) Examples of heterogeneous node density.

homogeneous, semi-homogeneous and heterogeneous, and we define them as follows:

- Homogenous distribution of the nodes: The neighbors are equidistantly positioned in rows and in columns in the $\mathrm{Ngh}$ [see Fig. 2(a)].

- Semi-homogeneous distribution of the nodes: A fixed number of rows and the distance between rows are defined. The same number of neighbors is distributed in each row where the neighbors are equidistantly positioned [see Fig. 2(b) and (c)].

- Heterogeneous distribution of the nodes: A fixed number of rows and columns and the distance between them are defined. We can have zones with different distribution of nodes into the Ngh [see Fig. 2(d)].

Depending on the scenario to be evaluated, a distribution model fits better than others. For example, a highway scenario is closer to a semi-homogeneous distribution than to a homogeneous one. Likewise, a cross street is closer to a heterogeneous distribution.

Finally, we consider the node mobility follows a straight line pattern. Furthermore, the relative distance between nodes A and B may change throughout the observation interval $T$.

\section{ANALYTiCAl MODEl For TRANSIENT ANALYSIS OF THE IDLE TIME}

In this section, based on the characterization of the packet traffic and the modeling of the link formed by two nodes, we propose a model for a transient analysis of idle time in a VANET. The notation used in our VANET modeling method is summarized in Tables I and II.
TABLE I

NOTATION AND SYMBOLS USED IN THE MODELING METHOD

\begin{tabular}{|c|c|}
\hline Link A-B & $\begin{array}{l}\text { The communication channel formed by the } \\
\text { nodes A and B. }\end{array}$ \\
\hline$T_{\text {idle_AB }}$ & Idle Time on the Link A-B. \\
\hline Ngh-A, Ngh-B & $\begin{array}{l}\text { Neighborhood of node A (resp. B). } \\
\text { (Set of nodes within its carrier sense range) }\end{array}$ \\
\hline JNgh-AB & Joint-Neighborhood of the Link A-B. \\
\hline$A c, B c, i A B$ & $\begin{array}{l}\text { Zone exclusive to A, exclusive to B, } \\
\text { common to both nodes (intersection), resp. } \\
\text { (The three zones form the JNgh-AB.) }\end{array}$ \\
\hline$T_{a}$ & $\begin{array}{l}\text { Interval between two consecutive channel } \\
\text { accesses by a node. }\end{array}$ \\
\hline$T_{u}, T_{s}$ & Using and silent period, resp., in a node. \\
\hline$\lambda_{g p}$ & $\begin{array}{l}\text { Packet generation rate to be } \\
\text { transmitted by a node. }\end{array}$ \\
\hline$T_{g p}=1 / \lambda_{g p}$ & $\begin{array}{l}\text { Time between two consecutive new packets } \\
\text { to be transmitted by a node. }\end{array}$ \\
\hline $\boldsymbol{u}$ state & $\begin{array}{l}\text { State 'transmitting a packet' of a node. } \\
\text { (The node is in a using period.) }\end{array}$ \\
\hline $\boldsymbol{n p}$ state, $\boldsymbol{c o}$ state & $\begin{array}{l}\text { State 'no packet' or 'contention', resp. } \\
\text { (The node is in a silent period.) }\end{array}$ \\
\hline$\underline{T_{n p}}, T_{c o}$ & Time that a node remains in a state. \\
\hline$\widetilde{T_{c o}}$ & Approximated value of $T_{c o}$ \\
\hline$\tau_{x}$ & Transmission time of a packet type $x$ \\
\hline$o_{x}$ & $\begin{array}{l}\text { Additional time using the channel because } \\
\text { of a packet transmission. } \\
\text { (Other time than packet transmission time.) }\end{array}$ \\
\hline$\Lambda$ & $\begin{array}{l}\text { Total packet load in an Ngh. } \\
\text { (Addition of } \lambda_{g p} \text { from all the nodes in an Ngh.) }\end{array}$ \\
\hline U-Load $=T_{u} \dot{\Lambda}$ & Load factor in a Ngh. \\
\hline$N^{A}$ & Quantity of nodes in Ngh-A. \\
\hline$N^{A c}, N^{B c}, N^{i A B}$ & $\begin{array}{l}\text { Quantity of nodes located in } \\
\text { each zone of JNgh-AB. }\end{array}$ \\
\hline$N_{u}^{A}, N_{n p}^{A}, N_{c o}^{A}$ & $\begin{array}{l}\text { Quantity of nodes in Ngh-A } \\
\text { staying in each state. }\end{array}$ \\
\hline$N_{\text {idle }}^{A}, N_{\text {busy }}^{A}$ & $\begin{array}{l}\text { Nodes in Ngh-A which sense } \\
\text { the medium as idle or busy, resp. }\end{array}$ \\
\hline$N_{c o}^{A}$ _enabled, $N_{c o}^{A}$-disabled & $\begin{array}{l}\text { Nodes in contention which sense } \\
\text { the medium as idle or busy, resp. }\end{array}$ \\
\hline$G_{A}=\left\{N_{u}^{A}, N_{n p}^{A}, N_{c o}^{A}\right\}$ & State of Ngh-A. \\
\hline $\begin{array}{l}M= \\
\left\{s p, G_{A c}, G_{i A B}, G_{B c}\right\}\end{array}$ & State of JNgh-AB. \\
\hline
\end{tabular}

TABLE II

VARIABLES FOR THE LOCATION AND MOVEMENT OF THE NODES

\begin{tabular}{ll}
\hline \hline A and B & $\begin{array}{l}\text { Nodes that define the Link A-B. } \\
D\end{array}$ \\
$d x, d y$ & $\begin{array}{l}\text { Distance between nodes A and B. } \\
\text { Horizontal and vertical distance, resp., } \\
\text { between any pair of adjacent nodes. }\end{array}$ \\
$r t x$ & $\begin{array}{l}\text { Transmission range. } \\
\text { (Maximum distance between nodes A and B.) }\end{array}$ \\
$r s n$ & $\begin{array}{l}\text { Carrier sense range. } \\
\text { (Maximum distance between a node and any }\end{array}$ \\
& $\begin{array}{l}\text { node in its Ngh.) } \\
\text { Number of nodes distributed in horizontal and }\end{array}$ \\
$V y$ & $\begin{array}{l}\text { vertical position, resp., in an Ngh. } \\
\text { Relative speed between nodes A and B. }\end{array}$ \\
$s p$ & $D$ converted into discretized value. \\
\hline \hline
\end{tabular}

\section{A. The Packet Traffic}

We are not interested in modeling the exact behavior of the traffic generated by a particular node, neither to differentiate if a packet transmission is or is not successful. Our interest is focused on knowing if no neighbor of Ngh-A and Ngh-B is using 


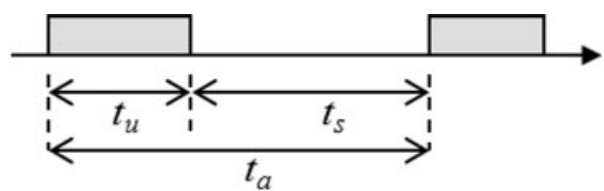

(a)

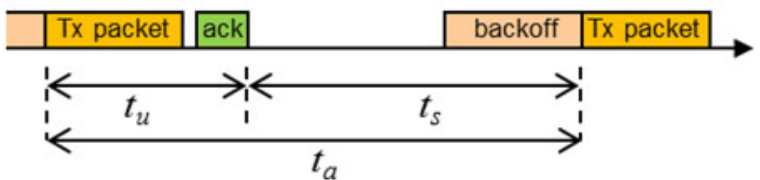

(b)

Fig. 3. Temporal diagram of a node occupying its communication channel. (a) Case of generic packet transmissions. (b) Case of unicast packet transmissions.

(or any neighbor of Ngh-A or Ngh-B is using) the communication channel formed by nodes A and B (i.e., Link A-B). This condition means that Link A-B is IDLE (or BUSY). Note that the reception at a neighbor node influences the channel occupancy of Link A-B if and only if this packet is also transmitted by a neighbor of node $\mathrm{A}$ or $\mathrm{B}$.

1) The Behavior of a Node: As a first step of modeling, we observe a particular node and identify when the node is or is not using the channel to transmit a packet. The node will stay in only one of two possible conditions: using the channel or being silent. Each use of the channel will be followed by a silence, forming this way a node access cycle which starts with a packet transmission. The access cycle of a node consists of a using period and a silent period (i.e., intervals in which the node is or is not using the channel, respectively). That cycle is repeated at each packet transmission. The duration of the using period mainly depends on the type of packet that is being transmitted (e.g., unicast data, hello messages), whereas the duration of the silent period depends on the packet transmission rate of the node and the channel condition (BUSY or IDLE).

Let us define three time intervals, which are drafted in Fig. 3(a). Let $t_{u}$ be the interval duration of the channel occupancy produced by a node (each time the node uses the channel), i.e., the duration of a using period; let $t_{s}$ be the interval since the moment when the use of the channel is over until the next access of the channel, i.e., the duration of a silent period; and let $t_{a}$ be the interval of time between two consecutive channel accesses. We have:

$$
t_{a}=t_{u}+t_{s}
$$

Let $T_{a}, T_{u}$ and $T_{s}$ be the mean values of $t_{a}, t_{u}$ and $t_{s}$, respectively. As each node can be in only one of the two periods (i.e., using period or silent period) at a given moment, we can define the using state and the silent state for a node. Let $\lambda_{u}$ be the transition rate from a using state to a silent state, and let $\lambda_{s}$ be the transition rate from a silent state to a using state. Consequently, each time a node visits a silent state or a using state, it remains in this state during an average period of $T_{s}=\frac{1}{\lambda_{s}}$ and $T_{u}=\frac{1}{\lambda_{u}}$, respectively. This is the simplest definition of states to differentiate whether a particular node is using the communication

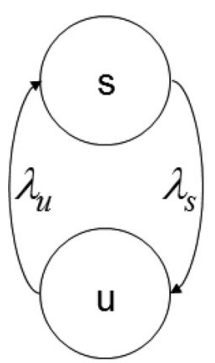

(a)

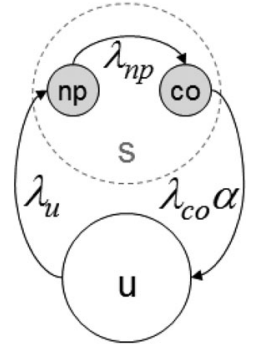

(b)
Fig. 4. States of the medium occupation by a particular node (s = silent state, $\mathrm{u}=$ using state). (a) States of the channel. (b) States of an individual node.

channel or not. A graphical representation of this definition is shown in Fig. 4(a).

We remind that we want to obtain a model as simple as possible while accurate enough to evaluate $T_{i d l e \_} A B$. However, this two states model can not be precise enough for our aims. The more quantity of nodes and the higher traffic load in the $\mathrm{Ngh}$, the less precise the results are. The reason is the influence of retransmissions and backoff stages. Modelling this influence over the transition from a silent state to a using state is a difficult task when considering a single state to model the silent period. Therefore, to differentiate a silent node being in contention state or not, we use two states instead of just a single state to model the silent period, as it is sketched in Fig. 4(b). In this figure, the state marked as np (no packet) corresponds to the node not being in transmission neither in contention, whereas the state marked as co (contention) corresponds to the node being in a contention stage before packet transmission. Consequently, the time elapsed in the np state and in the co state is equal to $T_{s}$, then:

$$
T_{s}=T_{n p}+T_{c o}
$$

Since we are not interested in studying the collisions nor the effective throughput, a differentiation between successful or unsuccessful packet transmissions is not needed in our model. This allows us to make some simplifications for our modeling as follows. Firstly, we model the time spent in contention with a single state. Hence, $T_{c o}$ represents the mean value of the total waiting time, in contention, before sending a packet. Conditioned to the fact that a node has a packet to transmit, $T_{c o}$ includes the time during which the node spends in DIFS, in backoff and also freezing backoff (i.e., the time during which the node senses the medium as busy).

In the literature, there are proposals which use more complex Markovian models to characterize the contention period, e.g., [24], [26]. Later, in Section V, numerical results will show that using a single state to model the time spent in contention period is enough to achieve our aim to evaluate $T_{i d l e}$ in the studied scenario. We assume that the $T_{c o}$ value is a priori known or periodically informed in the VANET. Later, to support this assumption, we will obtain a simple analytical expression of $T_{c o}$ that mainly depends on the load offered to the network (see Sections V-B and VI). 
Secondly, as the packet load increases on the communication medium, the nodes will experience an increment of the access rate due not only to new packets, but also to a higher number of retransmissions. In contrast, if a channel gets over-loaded until the point of becoming an unstable network, the access rate decreases because the packets experience long contention periods. Therefore, we could assume that the $T_{a}$ value will be a priori known or periodically informed in the VANET. Substituting (2) in (1), we obtain the staying time in np state:

$$
T_{n p}=T_{a}-T_{u}-T_{c o}
$$

Thirdly, a node being in the $\mathbf{u}$ state is transmitting a packet. A transition towards the $\mathbf{u}$ state will depend on whether any of its neighbors is or is not using the medium. This condition is addressed later in Section IV-A2. The mean value $T_{u}$ is calculated based on the mean transmission time of packets plus a constant value associated to the transmission of the packet:

$$
T_{u}=\sum_{\forall x} P_{x} \cdot\left(\tau_{x}+o_{x}\right)
$$

where $x$ identifies the packet type, e.g., unicast data, hello message; $P_{x}$ is the proportion of packets of type $x$ regarding the total number of packets; $\tau_{x}$ is the transmission time of a packet of type $x$, including its overhead (headers, preamble,...) and $o_{x}$ is the additional time occupying the channel due to the transmission of a type $x$ packet. For example, if the packet type is unicast data, an acknowledgement packet is always expected, which induces an extra time during which the channel remains in the using period. Fig. 3(b) shows an example of a unicast packet transmission, which implies the transmission of an ACK packet after a SIFS time. Therefore, we have at least $o_{d}=\operatorname{SIFS}+\tau_{A C K}$. In the case of hello packets (or any broadcast packet), no ACK packet is waited by the source node, the channel is only occupied during the packet transmission, so $o_{h}=0$, if the propagation time is negligible. In case of collision, a node can sense the channel as busy during a period longer than $\tau_{x}$ due to the overlap of collided packets. This condition is considered later in Section IV-A2.

Finally, we observe that when a node and the channel are not saturated, the mean access cycle is almost equal to the time between two new packets that the node wants to transmit, i.e., the packet generation interval. Let $\lambda_{g p}$ be the packet generation rate of the node, and $T_{g p}=\frac{1}{\lambda_{g p}}$.

To simplify our model, we can approximate $T_{a}$ such as $T_{a}=$ $T_{g p}$. Then, substituting this identity for $T_{a}$ in (3):

$$
T_{n p}=T_{g p}-T_{u}-T_{c o}
$$

Note that (5) could provide a negative value for $T_{n p}$. For instance, in case of a saturated network where nodes have always packets to transmit, due among other things to collisions, nodes will almost always be in $\mathbf{u}$ or co states and $T_{g p}$ is very likely smaller than $T_{u}+T_{c o}$. Then, we set $T_{n p}=0$ if (5) provides a negative value. It means that the model can be reduced to the model of Fig. 4(a) where $T_{s}=T_{c o}$. Later, in Section V-C, we will compare numerical results on $T_{i d l e}$ using both (3) and (5).

2) The Behavior of a Node's Ngh: We now characterize the behavior of the group of nodes belonging to an Ngh. For simplicity, we do not consider the different values of the parameters $T_{u}, T_{n p}$ or $T_{c o}$ that can be experienced by the different nodes, but we consider that $T_{u}, T_{n p}$ and $T_{c o}$ are mean values of the aggregated traffic in the Ngh (i.e., an average on all neighbors). On the other hand, if the localization and traffic of the nodes is highly heterogeneous, we can adapt the notation defined hereafter.

Let $N^{A}$ be the total number of neighbors in Ngh-A, where, at a given time, $N_{u}^{A}$ nodes are using the channel, $N_{c o}^{A}$ nodes are in contention and $N_{n p}^{A}$ nodes are not in contention nor using the channel, so $N^{A}=N_{u}^{A}+N_{n p}^{A}+N_{c o}^{A}$. The state of Ngh-A is defined as $G_{A}=\left\{N_{u}^{A}, N_{n p}^{A}, N_{c o}^{A}\right\}$.

In accordance with the previous definitions, $N_{u}^{A}=1$ is a sufficient condition for node A sensing the medium as busy (so node A will not start a transmission). However, the condition of having more than one node using the medium $\left(N_{u}^{A}>0\right)$ is also possible, because the distance between two nodes in Ngh-A can be longer than the carrier sensing range.

Let $\alpha$ be the factor that indicates if a node is or is not enabled to transmit, that is:

$$
\alpha= \begin{cases}1, & \text { if the node is enabled to transmit. } \\ 0, & \text { otherwise. }\end{cases}
$$

Then, in Ngh-A, we have that each one of the $N_{u}^{A}$ nodes leaves the transmitting period with a rate $\lambda_{u}$, each one of the $N_{n p}^{A}$ nodes transits to contention stage with a rate $\lambda_{n p}$ and each one of the $N_{c o}^{A}$ nodes leaves the silent period with a rate $\alpha \cdot \lambda_{c o}$.

The condition "the node is enabled to transmit" depends on the state of its neighbors. Note that some of its neighbors may not belong to Ngh-A or may not be a neighbor of some nodes in Ngh-A (i.e., the distance between them is longer than $r s n$ ). We use a simple approximation to estimate how many nodes are enabled to transmit in a Ngh as follows.

Let $N_{u}^{\max }$ be the maximum number of nodes that could be simultaneously transmitting in an Ngh. (For sake of simplicity, we have omitted any reference to node A.) This number is related to how the nodes in the Ngh area are geographically distributed. For example, in case of the left picture in Fig. 2(c), we estimate $N_{u}^{\max }=2$ in the worst case (e.g.,, the nodes at the rightmost and at the leftmost in the Ngh). Definitions in Section IV-B1 allow us to estimate $N_{u}^{\max }$. We now estimate how many neighbors sense the medium being idle or busy as a proportion of the number of nodes that are transmitting a packet. That is:

$$
\begin{aligned}
& N_{\text {idle }}^{A}=\operatorname{trunc}\left(\frac{N^{A} \cdot\left(N_{u}^{\max }-N_{u}^{A}\right)}{N_{u}^{\max }}\right) \\
& N_{\text {busy }}^{A}=N^{A}-N_{\text {idle }}^{A}
\end{aligned}
$$

Finally, we estimate also proportionally the number of nodes enabled or disabled to transmit. That is, the number of nodes in contention state is proportional to the number of nodes that sense the medium as idle or busy, respectively:

$$
\begin{aligned}
& N_{c o}^{A} \text { _enabled }=\operatorname{trunc}\left(\frac{N_{c o}^{A} \cdot N_{i d l e}^{A}}{N^{A}}\right) \\
& N_{c o}^{A} \text { _disabled }=N_{c o}^{A}-N_{c o}^{A} \text { _enabled }
\end{aligned}
$$




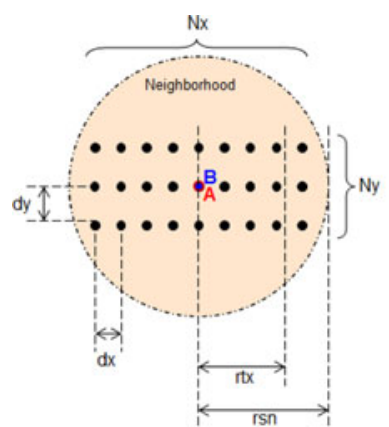

(a)

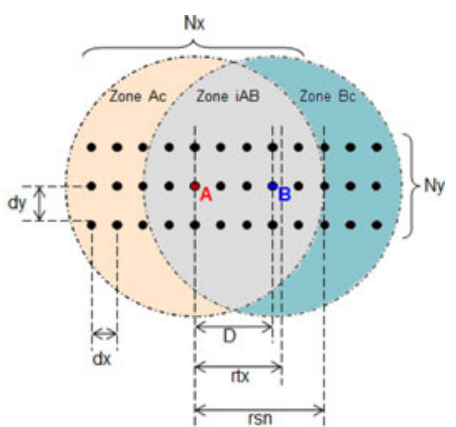

(b)
Fig. 5. Variables used in the model for the geographical distribution of the nodes in an Ngh and in an JNgh. (a) $D=0$. JNgh is a single Ngh. (b) $D>0$. JNgh, three zones.

To conclude, the possible transitions among the states of the Ngh-A, $G_{A}=\left\{N_{u}^{A}, N_{n p}^{A}, N_{c o}^{A}\right\}$, occur at the transition rates:

$\Lambda_{A} G^{-u}=N_{u}^{A} \cdot \lambda_{u}$, if a node stops its transmission;

$\Lambda_{A}^{G^{+u}}=N_{c o}^{A}$-enabled $\cdot \lambda_{c o}$, if a node begins to transmit;

$\Lambda_{A} G^{+c o}=N_{n p}^{A} \cdot \lambda_{n p}$, if a node wants to transmit.

Note that (9) are the transition rates due to a single node that changes its state. Therefore, Ngh-A also changes its state. For example, $\Lambda_{A} G^{-u}$ is the transition rate from the $\left\{N_{u}^{A}, N_{n p}^{A}, N_{c o}^{A}\right\}$ state to the $\left\{N_{u}^{A}-1, N_{n p}^{A}+1, N_{c o}^{A}\right\}$ state of Ngh-A, because of the event "one of the $N_{u}^{A}$ transmitting nodes stops to transmit".

\section{B. The Joint-Neighborhood (JNgh) of Link A-B}

In Section IV-A2, we have modeled the behavior of a single Ngh. However, to evaluate $T_{i d l e \_A B}$, the separate evaluations of Ngh-A and Ngh-B are not enough [9]. The Link A-B is actually IDLE when both nodes $\mathrm{A}$ and $\mathrm{B}$ are idle at the same time. Note that the only case in which Ngh-A and Ngh-B are the same neighborhood is when nodes $\mathrm{A}$ and $\mathrm{B}$ are in a geographical position that is almost the same [see the left image in Fig. 2(c)] and the distance between A and B (almost equal to 0) does not change throughout time.

1) Definition of the Joint-Neighborhood (JNgh) of Link A-B: To model the geographical location of the nodes that have an influence over Link A-B, we define different influence zones. The set of all the nodes within these different influence zones is what we call a Joint-Neighborhood (JNgh). Nodes influencing Link A-B belong to Ngh-A or to Ngh-B. Then, the JNgh for Link A-B (JNgh-AB) is formed by three different zones: nodes that belong to both neighborhoods (i.e., the intersection zone, $\boldsymbol{i A B}$ ), nodes that belong to Ngh-A but do not to Ngh-B (i.e., the exclusive zone of A, $\boldsymbol{A c}$ ) and nodes that belong to Ngh-B but do not to Ngh-A (i.e., the exclusive zone of B, Bc ). Note that in zones $A c$ and $B c$ we can find hidden nodes for nodes $\mathrm{B}$ and A, respectively. $T_{i d l e_{-} A B}$ is the cumulated time during which no node within JNgh-AB (i.e., zones $A c, i A B$ and $B c$ ) is using the medium. In Fig. 5(b) an example of a JNgh is shown. Red and blue nodes indicate nodes whose link is under analysis and therefore each one of them is the center of an $\mathrm{Ngh}$ of interest. In this example, Ngh-B has the same node density and radius as Ngh-A.

For simplicity, and since in the present work we will evaluate a highway scenario, we hereafter expose the generic modeling using a semi-homogeneous distribution of the nodes. Cases of more complex densities can also be analyzed with our methodology, by extending this notation. For example, Fig. 2(d) show different node densities for each zone $A c, i A B$ and $B c$. In this case, even the nodes' distribution could change during $T$ in a way that evaluations in subintervals of $T$ can be considered.

In Table II, the variables that define the location and movement of the nodes are summarized.

Fig. 5(a) shows some of the variables defining the geographical distribution of the nodes in an Ngh. In the example of Fig. 5(a), Ngh-A has 3 lanes $(\boldsymbol{N y}=3)$ with a distance $d y$ among them. In the lanes, all the nodes are equidistantly distributed in the Ngh. It means that the total distance in the $x$ axis is approximately equal to (applying Pythagoras' theorem):

$$
\left(N_{x}-1\right) \cdot d x=\sqrt{(2 \cdot r s n)^{2}-\left(\left(N_{y}-1\right) \cdot d y\right)^{2}}
$$

Furthermore, in the example of Fig. 5(a), we have 9 nodes per lane $(\boldsymbol{N} \boldsymbol{x}=9)$. Then, the distance $\boldsymbol{d} \boldsymbol{x}=$ $\sqrt{(2 \cdot r s n)^{2}-(2 \cdot d y)^{2}} / 8$.

We model the mobility of nodes A and B by the relative distance between both nodes, denoted as $D$. $D$ has a clear influence on the size of the zones $A c$ and $B c$ and therefore on the amount of nodes within JNgh-AB. In addition, the area of each $\mathrm{Ngh}$ is approximated as a square area.

Besides, in the scenarios evaluated in this work, the node motion follows a lane. Then, we have a two dimensional plane where the different lanes are placed in the $y$ axis and the movement of nodes A and B follows the $x$ axis. Moreover, we assume that the density of nodes within the JNgh has the same pattern throughout time (i.e., a moving vehicle always has the same density of nodes in its JNgh). For example, Fig. 5(a) and (b) show such a case where the distance between nodes $\mathrm{A}$ and $\mathrm{B}$ is $D=0$ and $D>0$ respectively, and the node density within $\mathrm{JNgh}-\mathrm{AB}$ is the same.

In our scenario, we assume that the link is never broken during the observation interval $T$ to evaluate $T_{i d l e_{-} A B}$, thus $D \leq r t x$. The evolution of $D$ through interval $T$, and consequently the evolution of the quantity of nodes in the $A c$ zone and the $B c$ zone, depends on the relative speed between nodes $\mathrm{A}$ and $\mathrm{B}$ (i.e., $\boldsymbol{V}$ ). Note that, in our model, a change in the distance value $D$ means a change in the zones $A c, i A B$ and $B c$. Each different value for $D$ being in $d x$ units closer or farther between $\mathrm{A}$ and $\mathrm{B}$ means a difference in the quantity of nodes within JNgh-AB. Consequently, discrete steps in $d x$ can be considered to model the node movement. If $V \neq 0$, a change in the number of nodes (in JNgh-AB and in each one of its zones) takes place each $\left|\frac{d x}{V}\right|$ time unit, or never if $V=0$.

Let $s p$ be the distance between A and B converted into discretized values (steps of $d x$ value). Then, $s p=\left\lceil\frac{D}{d x}\right\rceil$ and $s p$ changes are produced at a rate $\lambda_{s p}=\left|\frac{V}{d x}\right|$. 


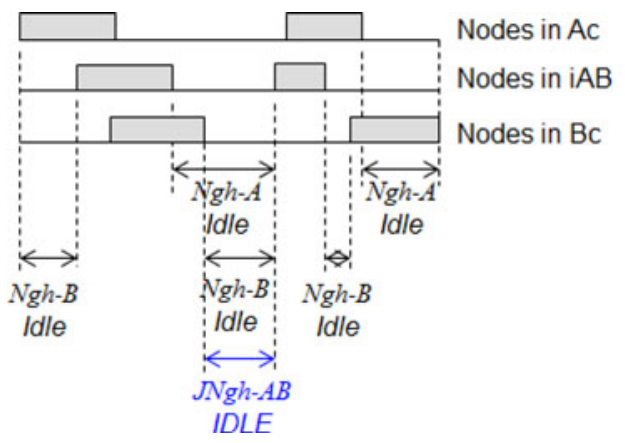

Fig. 6. Temporal diagram of the different nodes that occupy the communication channel for the different zones in a JNgh.

2) The Behavior of the JNgh of Link A-B: According to our JNgh-AB definition, the Link A-B is IDLE when all the zones in JNgh-AB ( $i A B, A c$ and $B c)$ are simultaneously idle. An example of the channel use in the different zones are given in Fig. 6. Each temporal axis shows if at least one node in each zone of JNgh-AB is using the channel. This diagram also indicates the intervals sensed as busy or idle by nodes A and B.

We now extend the notation to identify nodes belonging to the different zones $(A c, B c$ and $i A B)$, and also the node states and their influence on the state of Link A-B. We handle the zones JNgh-AB as if each one of the zones would be a single $\mathrm{Ngh}$. That is, each JNgh-AB zone is identified by each state $G_{A c}, G_{i A B}$ and $G_{B c}$, as it is defined in Section IV-A2. In addition, we incorporate the modeling of the relative movement between nodes $\mathrm{A}$ and $\mathrm{B}$, which will modify zones $A c$ and $B c$, as it is described in Section IV-B1. That is, the value $s p$ (in step units) defines the state of the distance between A and B, and consequently the number of nodes being in each zone, i.e., $N^{A c}$, $N^{i A B}$ and $N^{B c}$.

Let $M=\left(s p, G_{A c}, G_{i A B}, G_{B c}\right)$ be the state of JNgh-AB. The state transitions of $M$ will be due to the transition of one node of JNgh-AB or a change in the distance between nodes A and $\mathrm{B}$. Thus, any state change of $M$ is produced by any state change of $G_{A c}, G_{i A B}, G_{B c}$ or $s p$. Note that when nodes $\mathrm{A}$ and $\mathrm{B}$ are very close $(s p=0)$, it is a particular case of $M$, where only $G_{i A B}$ will exist (all the nodes of JNgh-AB are in the zone $\left.G_{i A B}\right)$, i.e., $N^{A c}=0, N^{i A B}=N$ and $N^{B c}=0$, where $N$ is the number of nodes in JNgh-AB.

Finally, the Link A-B is IDLE if and only if none of the nodes is using the medium, i.e., $N_{u}^{A c}=0, N_{u}^{i A B}=0$ and $N_{u}^{B c}=0$; otherwise, it is BUSY.

Summarizing, to calculate $T_{i d l e_{-} A B}$ we count the time in which both nodes, A and B, simultaneously experiment an idle interval. $T_{i d l e_{-} A B}$ is the cumulated time in which no node within JNgh-AB is using the medium.

\section{Evaluation of the Model}

Let $\chi=\{X(t): t \geq 0\}$ be a continuous time Markov chain (CTMC) that models the JNgh-AB behavior: the CTMC states are the states $M$ defined in Section IV-B2. Let $\mathbf{r}$ be the vector of rewards, whose component $r_{j}$ defines the reward associated to state $j$. Then, $r_{X(t)}$ is the reward experienced in the system at time $t$. The CTMC with its associated rewards defines a Markov reward chain (MRC). Let $R(t)$ be the random variable that characterizes the total reward accumulated during a measurement period $t$ in the $\mathrm{JNgh}-\mathrm{AB}$, i.e.,:

$$
R(t)=\int_{o}^{t} r_{X(s)} d s
$$

In the performability research area, $R(t)$ is the most employed random variable to characterize the system efficiency. In the particular case of rewards that only get values 0 or 1 , the PDF evaluation of $R(t)$ is known as dependability [27]. This name is due to the fact that these reward values can be interpreted as states of the system in failure (i.e., $r=0$ ) or in full operation (i.e., $r=1$ ). In this case, $R(t)$ corresponds to the total time in which the system is available during the interval $[0, t]$. Accordingly, we obtain $T_{i d l e_{-} A B}$ when we evaluate the first moment of the random variable $R(t)$, where each reward $r_{j}$ is value 0 or 1 for the state $j$ being BUSY or IDLE, respectively, in the Link A-B. We remind that Link A-B is IDLE if and only if none of the neighbor nodes is using the medium, i.e., $N_{u}^{A c}=0, N_{u}^{i A B}=0$ and $N_{u}^{B c}=0$; otherwise, it is BUSY (see Section IV-B2).

\section{NumERICAL EVALUATION}

The implementation to obtain the numerical evaluation from the MRC has been carried out using the algorithm proposed in [28]. On the other hand, to test the accuracy of our proposed model, simulations were performed using the NCTUns 6.0 network simulator [29]. All our simulation code for NCTUns is available at [30].

\section{A. Simulated VANET Configuration Parameters}

Table III shows the most important parameters used to configure the VANET in the simulator. Seven cases with different data packet loads generated by the vehicles of the VANET are studied. We refer to them in Table III as case 1 to case 7. Also, in each case, different vehicles in the scenario have different packet loads. Each vehicle sends different packet types (unicast and broadcast packets).

All vehicles move on two lanes and in the same direction, with speeds bounded by a minimum and maximum value, mainly due to the vehicles density. In the considered highway, the distribution of nodes is almost uniform. In addition, all nodes have the same transmission range and carrier sensing range, which are $200 \mathrm{~m}$ and $300 \mathrm{~m}$, respectively. The number of vehicles within the carrier sensing area of vehicles A or B changes throughout simulation time, although it is limited to a maximum of 15 vehicles.

\section{B. Parameter Calculation for the Analytical Model According to the VANET Configuration}

1) Computation of the Model Parameters: For the VANET scenario depicted in Section III with the specific values specified in Section V-A, we now establish which are the model parameter values that represent the best this VANET scenario. 
TABLE III

CONFIGURATION AND SimUlation SETTINGS OF THE VANET SCENARIO

\begin{tabular}{|l|l||l|l|}
\hline MAC layer protocol & IEEE 802.11p & Slot time & $13 \mu \mathrm{s}$ \\
MAC header & 28 bytes & DIFS & $58 \mu \mathrm{s}$ \\
PREAMBLE time & $32 \mu \mathrm{s}^{*}$ & SIFS & $32 \mu \mathrm{s}$ \\
SIGNAL time & $8 \mu \mathrm{s}$ & $r t x$ & $200 \mathrm{~m}$ \\
SERVICE+Tail bits & 24 bits & $r s n$ & $300 \mathrm{~m}$ \\
\hline Bandwidth & & $6 \mathrm{Mbps}$ \\
\hline Number of nodes: & Total in the scenario & 100 nodes \\
& Max. within $r$ sn area & 15 nodes \\
& Max. within $r t x$ area & 11 nodes \\
\hline Highway: & Length & $4.7 \mathrm{~km}$ \\
& Lanes & 2 \\
& Width & $12 \mathrm{~m} / \mathrm{lane}$ \\
\hline Speed of the cars: & Max. & $30.56 \mathrm{~m} / \mathrm{s}$ \\
& Min. & $13.8 \mathrm{~m} / \mathrm{s}$ \\
\hline Hello packet: & Size & $1 \mathrm{pk} / \mathrm{s}$ \\
\hline & Rate & Unicast \\
& & Exponential \\
\hline Data packet: & Type \\
& Distrib. & & $100 / 500 / 1400$ bytes \\
\hline
\end{tabular}

Packet generation rate at each node:

Distrib. $\quad$ Exponential with different Min. /Average /Max. values $\quad$ Case 1 to case 7

* Corresponding to the transmission of 2 long symbols (at $8 \mu$ s each one) and 10 short symbols (at $1.6 \mu$ s each one).
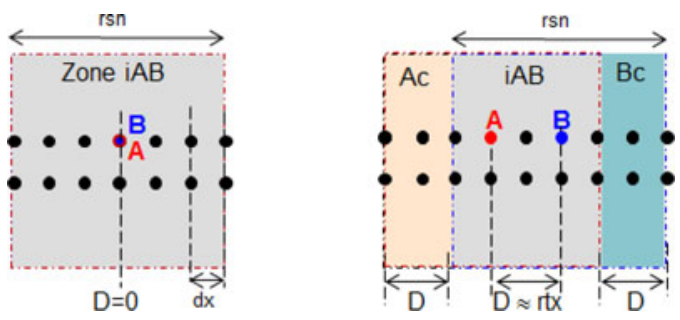

Fig. 7. The node distribution model for the evaluated VANET scenario.

We apply a semi-homogeneous geographical model for the locations of nodes. We estimate that the total number of nodes into an Ngh is 14 nodes, which are distributed in the 2 lanes with 7 vehicles per lane. Moreover, this node distribution is almost constant along road and thus through time. Then, the total number of nodes that influence the Link A-B (i.e., within the JNgh- $\mathrm{AB}$ ) will be approximately 14 nodes at the minimum distance $(D=0 \mathrm{~m})$ and 18 nodes at the maximum distance $(D \approx r t x=200 \mathrm{~m})$ (see left and right pictures, respectively, in Fig. 7). Note that, since $r s n$ is the same value for both nodes, the width (i.e., the distance in the axis $x$ ) of each zone $A c$ or $B c$ is equal to $D$.

For each case (case 1 to case 7), we calculate the total packet load in an Ngh, denoted as $\Lambda$ (see Table IV). $\Lambda$ is the sum of the packet rates of all the nodes in an Ngh. We consider that nodes are homogeneous in the sense that the packet load is equal in each node, so the traffic generated by each node $\lambda_{g p}$ is equal to $\Lambda$ divided by the total number of nodes into the Ngh. Note that, in general, $\Lambda$ and $\lambda_{g p}$ could be different in other more complex scenarios. In this work we consider that $\Lambda$ and $\lambda_{g p}$ have the same values in Ngh-A and Ngh-B, and let the analysis of the heterogeneous case for future work.
TABLE IV

EXPERIMENTAL CONTENTION PERIOD $\left(T_{c o}\right)$, APPROXIMATE CONTENTION PERIOD $\left(\widetilde{T_{c o}}\right)$, Estimated TOTAL PACKET LOAD IN AN NGH $(\Lambda)$ AND FACTOR U-LOAD IN AN NGH

\begin{tabular}{lcccc}
\hline \hline Configuration & $\Lambda(\mathrm{pk} / \mathrm{s})$ & $\widetilde{T_{c o}}(\mu \mathrm{s})$ & $T_{c o}(\mu \mathbf{s})$ & U-Load \\
\hline case 1 & 47.668 & 52.131 & 109.379 & 0.0351 \\
case 2 & 182.342 & 162.304 & 155.422 & 0.1645 \\
case 3 & 350.684 & 300.020 & 242.397 & 0.3264 \\
case 4 & 550.200 & 463.240 & 421.397 & 0.5182 \\
case 5 & 737.870 & 616.768 & 629.498 & 0.6986 \\
case 6 & 865.612 & 721.270 & 753.644 & 0.8214 \\
case 7 & 1086.400 & 901.892 & 905.881 & 1.0336 \\
\hline \hline
\end{tabular}

TABLE V

PARAmeters of the ANALYTICAL Model

\begin{tabular}{|l|l||l|l|}
\hline$N$ & 14 nodes & $d y$ & $12 \mathrm{~m}$ \\
$N y$ & 2 nodes & $d x$ & $99.98 \mathrm{~m}=1$ step \\
\hline$\lambda_{u}$ & 0.01767367 1/slot & $\lambda_{c o}$ & 0.11885280 1/slot \\
$\lambda_{s p}$ & 0.00000062 step/slot & $\lambda_{n p}$ & 0.00004439 1/slot \\
\hline Max. steps & 2 steps \\
\hline$M_{\text {initial }}=\left(s p, G_{A c}, G_{i A B}, G_{B c}\right)$ \\
$s p$ & 2 \\
$G_{A c}=\left(N_{u}^{A c}, N_{n p}^{A c}, N_{c o}^{A c}\right)$ & $(0,4,0)$ \\
$G_{i A B}=\left(N_{u}^{i A B}, N_{n p}^{i A B}, N_{c o}^{i A B}\right)$ & $(0,6,0)$ \\
$G_{B c}=\left(N_{u}^{B c}, N_{n p}^{B c}, N_{c o}^{B c}\right)$ & $(0,4,0)$ \\
\hline
\end{tabular}

Rewards $r_{M}$

$$
\begin{array}{ll}
1 & , \text { if } N_{u}^{A c}=N_{u}^{i A B}=N_{u}^{B c}=0 . \\
0 & , \text { otherwise. }
\end{array}
$$

Values for the VANET configured according to case $3, V=4.8 \mathrm{~m} / \mathrm{s}$ and $D=190 \mathrm{~m}$

We compute $T_{u}$ as a weighted average of all the packet traffic in an Ngh, using (4). In that equation, according to Table III, the transmission time $\left(\tau_{x}\right)$, for data and hello packets, are $864 \mu \mathrm{s}$ and $192 \mu \mathrm{s}$, respectively, and the time $o_{\text {data }}$ is $96 \mu \mathrm{s}$.

In our modeling, we assume that the $T_{c o}$ value is known. Thus, for the numerical results in this section we consider an estimation of the mean contention period that we denote as $\widetilde{T_{c o}}$. Table IV shows $\widetilde{T_{c o}}$ (converted to units $\mu$ s) values for each load case. These values are obtained (in slot units) as:

$$
\widetilde{T_{c o}}=65.31 * \mathrm{U}-\text { Load }+1.77
$$

where U-Load is a load factor that allows us to consider the influence of both packet rate and packet transmission time over $T_{c o}$, in the following way:

$$
\mathrm{U} \text {-Load }=T_{u} \cdot \Lambda
$$

Equations (12) and (13) were empirically obtained. Nonetheless, those equations can be explained by the fact that the contention periods of a node depend on the traffic to transmit on the medium and also on the transmission time required by each packet that uses the medium. In section VI we will present different results for $T_{c o}$ and $\widetilde{T_{c o}}$ to show that these equations are generic enough by considering other VANET scenarios different from the ones under analysis in this work.

2) Example of Parameter Values in a Specific Case: Table V gives the values of the different parameters used in the analytical 
model in case 3 , with a relative speed $V=4.8 \mathrm{~m} / \mathrm{s}$ and an initial distance between nodes A-B $D=190 \mathrm{~m}$. These values are obtained as follows. The lane width is $12 \mathrm{~m}$ in our scenario, so $d y=12 \mathrm{~m}$ in the $y$ axis. There are 2 lanes, so $N y=2$, and 7 vehicles in each lane, so $N x=7$. In addition, $r s n$ is $300 \mathrm{~m}$. Then, applying (10), 7 vehicles are uniformly distributed in about $599.88 \mathrm{~m}$ through the $x$ axis and, hence the step $d x$ (that is also the distance between adjacent nodes in a lane) is equal $99.98 \mathrm{~m}$. rtx is $200 \mathrm{~m}$ so nodes A and B can be far away of at most 2 steps of distance. Then, for a speed $V=4.8 \mathrm{~m} / \mathrm{s}$, a step can be traveled after $20.8292 \mathrm{~s}$. Using (4) and (5) we obtain $T_{u}$ and $T_{n p}$, respectively, where $T_{g p}=39.922 \mathrm{~ms}$ and $T_{c o}=$ $300.020 \mu \mathrm{s}$ is obtained through (12). Finally, we convert all the values into step units and slot units. Note that any value $D$ in our model is discretized according to this step, as it was modeled in Section IV-B1. That means that the distance values will be near to ceiling $(D / d x)$. Therefore, the distances $D=0,0<$ $D \leq 99.98$ and $99.98<D \leq 200$ provide the three possible different values for $s p$ (that is, step 0,1 and 2, respectively) for a VANET configured according to Table III. Obviously, the modeling of other VANET configurations with a higher density of nodes in each lane will have a lower $d x$ value and therefore a higher value of maximum quantity of steps.

Values in Table $\mathrm{V}$ are the input parameters of a function to constitute the transitions matrix of the JNgh- $\mathrm{AB}$ that represents the evolution of the Link A-B in the VANET. Finally, once the generating matrix and the rewards created, we obtain the MRC which is evaluated using the method pointed out in Section IV-C.

As a conclusion, using nominal values of a VANET configuration (see Table III), we can estimate the values of the parameters of the analytical model for the VANET scenario (e.g., see Table V). Note that if we were evaluating a real VANET online, we could have, at disposal more accurate values to be considered in the modeling parameters, e.g., $T_{c o}$ measurements. Also, we remind that during the VANET operation, it is not enough that nodes compute the idle time in their Ngh, since we furthermore need to know when the Ngh of both link nodes are simultaneously idle. Furthermore, we would like to highlight that the particular VANET scenario evaluated in this section satisfies a twofold requirement. On the one hand it is simple enough to develop our analysis; and on the other hand it is general enough to represent most of the scenarios we may found in a VANET. The only limitation, for the sake of a simple model, concerns the homogeneity assumption. Nevertheless, as pointed out in Section VII we plan to tackle the heterogeneous case in a near future.

In the following, we will compare both simulation and analytical results to evaluate the accuracy of our model. Note that, regarding simulations, we simulate a realistic VANET where the backoff evolves according to collisions, channel errors, among other factors.

\section{Obtained Results}

At the beginning of an observation interval $T$, the distance $D$ and the relative speed $V$ between nodes A and B selected in the simulations are considered as the initial state of the VANET.
TABLE VI

$T_{i d l e}$ Numerical Results from Model vs. Simulation Results

\begin{tabular}{lccc}
\hline \hline Case & SIM & MODEL & Relative error $(\% T)$ \\
\hline case 1 & 0.9601 & 0.9565 & 0.0036 \\
case 2 & 0.8134 & 0.7963 & 0.0171 \\
case 3 & 0.6398 & 0.6018 & 0.0380 \\
case 4 & 0.4165 & 0.3968 & 0.0196 \\
case 5 & 0.2276 & 0.2563 & 0.0286 \\
case 6 & 0.1823 & 0.1965 & 0.0142 \\
case 7 & 0.1745 & 0.1447 & 0.0298 \\
\hline \hline
\end{tabular}

Example for $T=25 \mathrm{~s}, D=190 \mathrm{~m}$ and $V=4.8 \mathrm{~m} / \mathrm{s}$

In each studied case 1 to 7 , we run a whole simulation (200 s) instead of several simulations with different values of $D$ and/or $V$ for each different observation interval $T$. With a single long simulation, the values of $D$ and $V$ will also vary according to time. For a given $T$, we record the system initial state and calculate diverse measures by simulation and with our model. This way, we get simulation results under different conditions of the system and we can compare them with the results from our model. Note that our model approximates the variation of distance between nodes in discretized values $(s p)$. We remind that the velocity $V$ is the relative speed between two vehicles (nodes A and B), which we consider to be constant during $T$. Therefore, we highlight that the following figures show the relative speed $V$ and not the individual speed of the vehicles in the highway.

1) Impact of the Packet Generation Rate: Fig. 8 presents simulation results on the mean idle time $T_{i d l e_{-} A B}$ observed on the studied link according to the total packet load ( $\Lambda$, see Table IV). Fig. 8(a) and (b) show the evaluations for each interval of $T=10 \mathrm{~s}$ and $T=25 \mathrm{~s}$, respectively. In Fig. 8 the exact points obtained with different loads (case 1 to case 7 ) are depicted. We also show the trend line for the representative cases of minimum, middle and maximum initial distance $(D)$. The packet load offered to the network is one of the most influencing parameters in the VANET performance. In the different cases, we can see that the higher the load, the lower $T_{i d l e \_} A B$. The minimum $T_{i d l e} A B$ is about $15 \%$ of $T$, which is achieved with a total load of $800 \mathrm{pk} / \mathrm{s}$ (approximately) or higher. Moreover, in Fig. 8, we can notice that the higher influence of $D$ is achieved when the load is medium (around $600 \mathrm{pk} / \mathrm{s}$, where the VANET is not overloaded neither under-loaded). In this case, the diversity in terms of competition for the VANET communication medium is wider and then the influence of the hidden nodes on $T_{i d l e \_A B}$ can be higher than $10 \%$ for the evaluated VANETs. On the other hand, in the lowest and the highest values of load, for the different values of $D$ and $V$, the dispersion of $T_{i d l e \_} A B$ is minimal. Also, less dispersion in the average values is expected as the evaluation interval $T$ increases.

In Fig. 9, we present the results for $T_{i d l e_{-} A B}$ of both modeling and simulation approaches for $T=25 \mathrm{~s}$. Each picture corresponds to different values for $D$ and $V$. We can observe that for all the evaluated cases, the results obtained from the analytical model fit well with the ones obtained from simulation. Besides, in Table VI we show $T_{i d l e_{-} A B}$ values obtained for each 


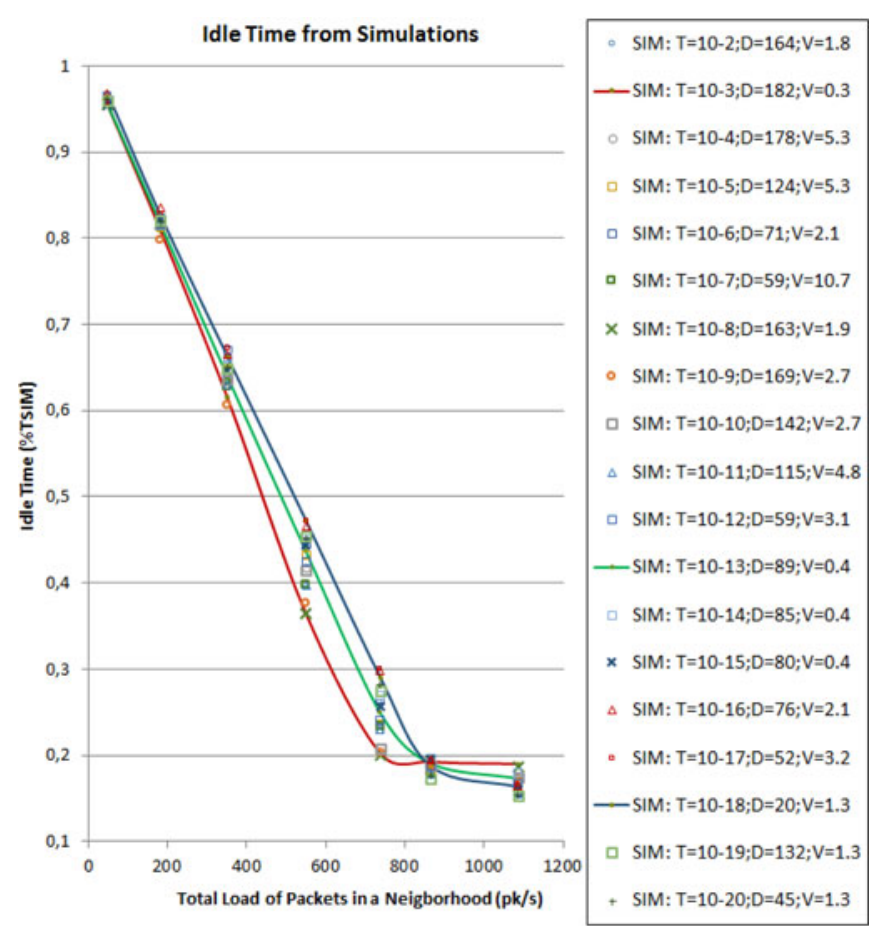

(a)

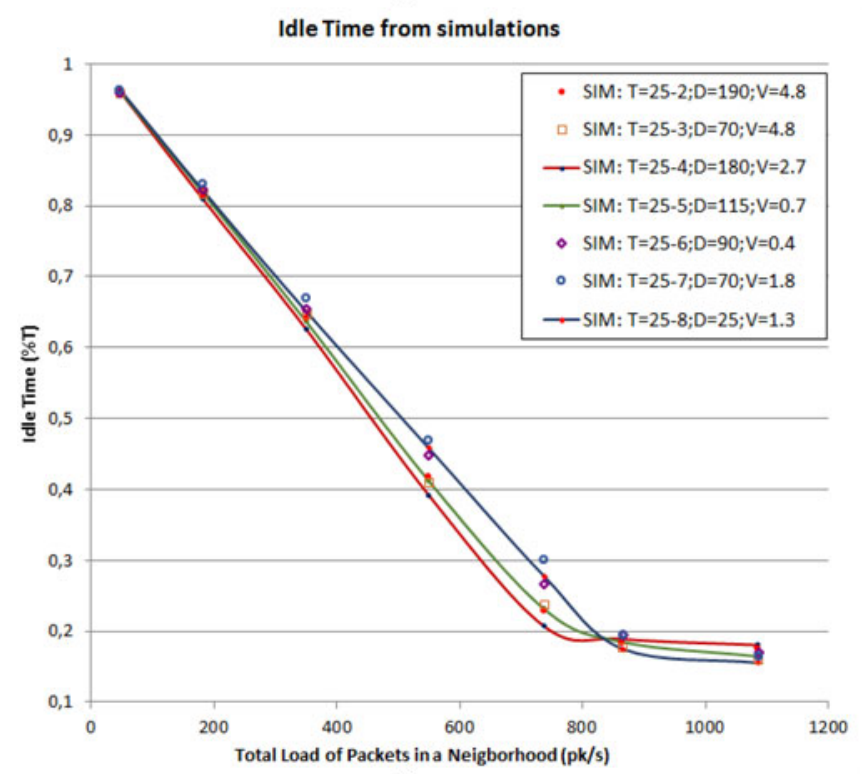

(b)

Fig. 8. Simulation results on the mean idle time $\left(T_{i d l e \_} A B\right)$ with different values on the initial distance $(D)$ and relative speed $(V)$. (a) Evaluations for intervals $T=10 \mathrm{~s}$. (b) Evaluations for intervals $T=25 \mathrm{~s}$.

load case (case 1 to case 7) for the particular interval $T=25 \mathrm{~s}$, with $D=190 \mathrm{~m}$ and $V=4.8 \mathrm{~m} / \mathrm{s}$. In addition, we indicate the relative error ( $\%$ of $T$ ) between the simulated and the analytical values, which shows a reasonable accuracy level. In this example $(T=25 \mathrm{~s}, D=190 \mathrm{~m}$ and $V=4.8 \mathrm{~m} / \mathrm{s})$ we can see that the error is lower than $3.8 \%$ of $T$. Also, the error is moderate for case 5 and case 7 in which the relative error is $2.86 \%$ of $T$ and $2.98 \%$ of $T$, respectively. In all the other examples that we have carried out, the maximum error was $6.3 \%$ of $T$.
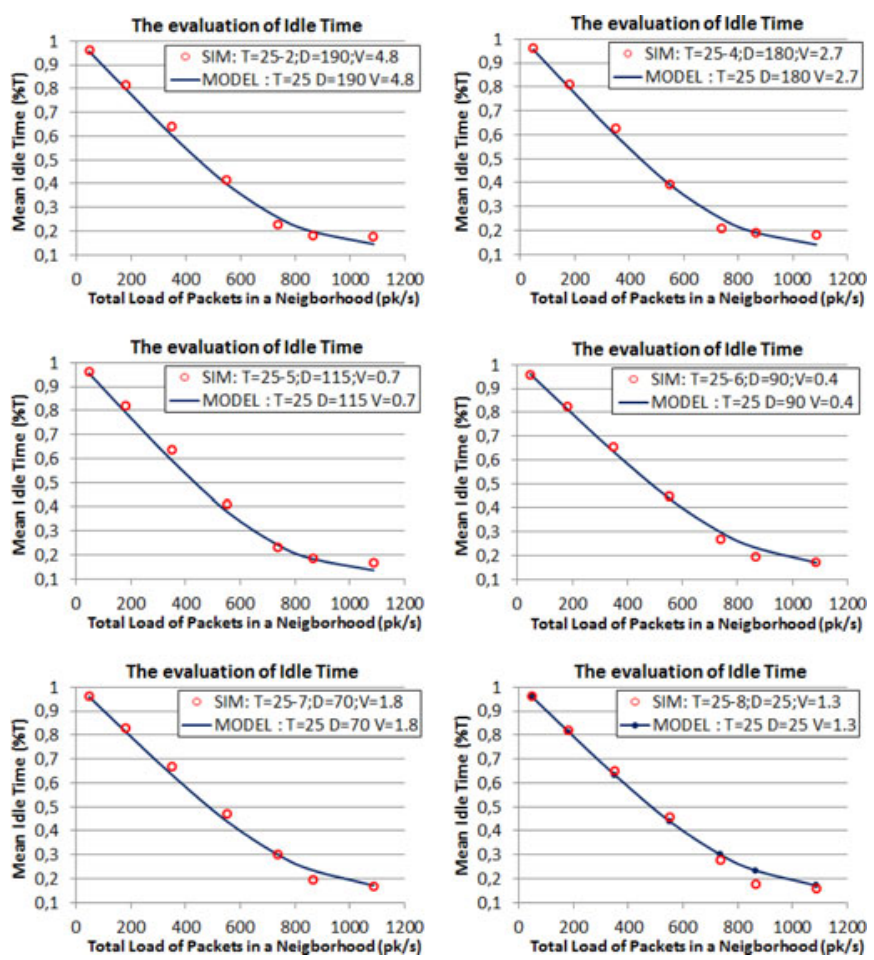

Fig. 9. Numerical results of $T_{i d l e}$ from the model vs. simulation results.

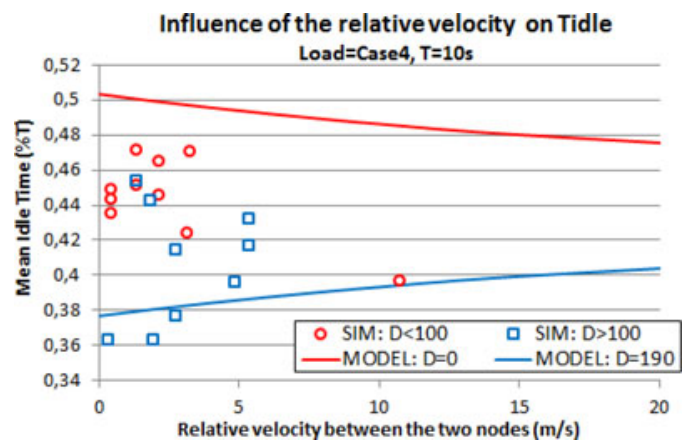

Fig. 10. Influence of the relative speed $(V)$ on $T_{i d l e} A B$. Example for a VANET scenario configured as case 4 with an observation interval $T=10 \mathrm{~s}$.

2) Impact of the Relative Velocity and the Initial Distance: The impact of $D$ and $V$ are shown in Figs. 10 and 11(a). The influence of $V$ and $D$ is jointly shown in Fig. 10, which displays the evaluation of $T_{i d l e \_A B}$ as a function of the $V$ value. In Fig. 10, the results are classified in cases where short and long initial distance happen, corresponding to $D<100 \mathrm{~m}$ and $D>100 \mathrm{~m}$ for simulation results, and $D=0 \mathrm{~m}$ and $D=190 \mathrm{~m}$ for the results of the model. We can observe that a higher value of $T_{\text {idle_AB }}$ is obtained for cases with lower $D$, in contrast to the lower values of $T_{i d l e_{-} A B}$ if $D$ values are high. Moreover, as speed increases, the results where the distance is short $(D<100$ m) are more similar to the ones where the distance is long $(D>100 \mathrm{~m})$. That means that the results of the cases with short and long distances are becoming much similar because a higher mobility of the nodes causes a faster change of the distance between the nodes and thus reduces the influence of the initial distance on $T_{i d l e \_A B}$. 


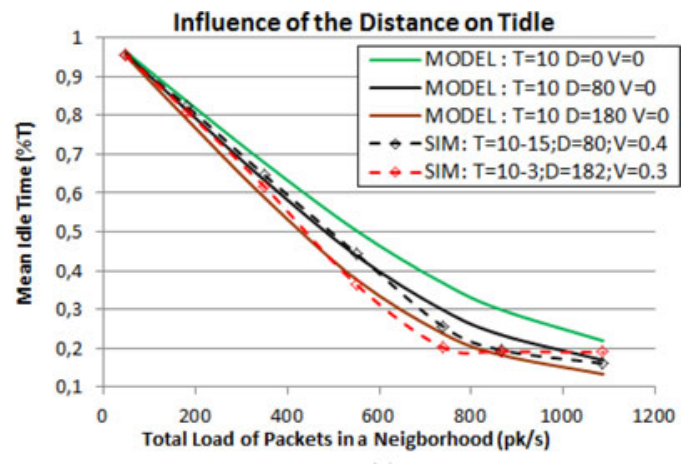

(a)

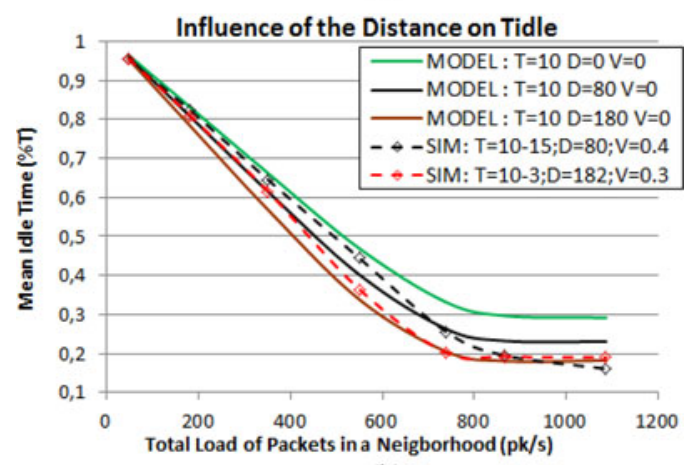

(b)

Fig. 11. Comparative results on $T_{i d l e \_A B}$ for different values of $D$. (a) Modeling with simplified parameter $T_{a}=T_{g p}$ (5). (b) Modeling with experimental parameter $T_{a}$ (3).

To conclude, as we expected, the higher the speed $V$, the less influence of the initial distance $D$ on the $T_{i d l e}$ results. This happens because vehicles at high speeds quickly change of positions which makes the distance during an observation interval varying and different from the distance at the beginning of the observation interval. In contrast, when the speed $V$ equals 0 , the distance $D$ will be maintained during the whole interval $T$, and therefore the maximum influence of $D$ will be obtained on the mean idle time. Those cases with the bigger influence of $D$ (i.e., for $V=0 \mathrm{~m} / \mathrm{s}$ ) are shown in Fig. 11(a). With an initial distance that is minimum $(D=0 \mathrm{~m})$, middle $(D=80 \mathrm{~m})$ and maximum $(D=180 \mathrm{~m})$, the step $s p$ is equal to 0,1 and 2 , respectively, which are the three possible distances modeling this particular scenario. Also, Fig. 11(b) depicts two representative simulation cases. They have a low speed $(V=0.3 \mathrm{~m} / \mathrm{s}$ and $V=0.4 \mathrm{~m} / \mathrm{s})$, and an initial distance that is middle $(D=80 \mathrm{~m})$ and long $(D=182 \mathrm{~m})$. We can see a similar tendency in terms of results from the model and from the simulations regarding the values of $D$ and load. As it was also expected, the influence of the distance on $T_{i d l e \_A B}$ shows that the longer the distance, the lower $T_{i d l e \_} A B$. This is because the higher the value of $D$, more nodes have an influence on Link-AB, i.e., the larger is the area of the JNgh defined by nodes A and B.

In order to check if the model can provide more accurate results when more precise $T_{a}$ values are considered, Fig. 11(b) presents the model evaluation using the $T_{a}$ values experimentally obtained from our simulations instead of $T_{a}=T_{g p}$ (see (3)). We observe that $T_{\text {idle }}$ results from the model are less precise for middle packet loads. For example, we can see in

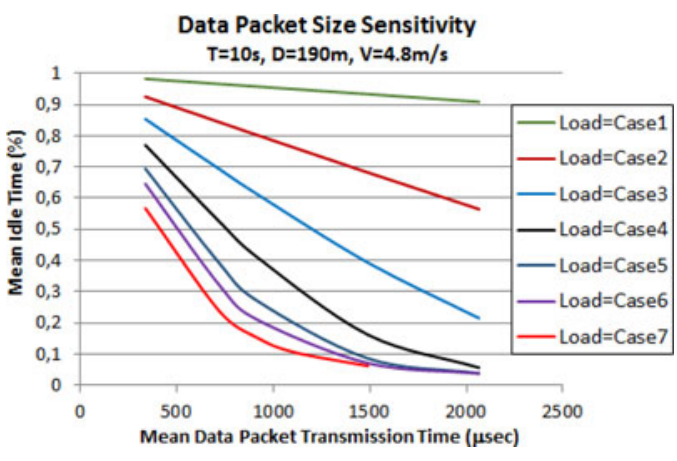

Fig. 12. $T_{i d l e_{A} A B}$ results from the model for different packet sizes.

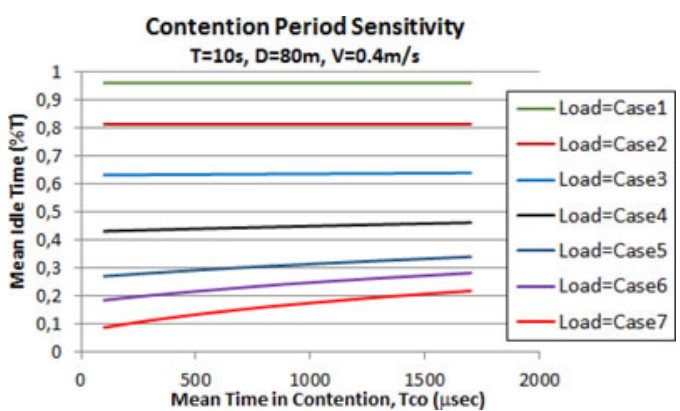

Fig. 13. $T_{i d l e \_} A B$ results from the model for different values of $\widetilde{T_{c o}}$.

Fig. 11(a) that simulation results for some load points (approximately, from $500 \mathrm{pk} / \mathrm{s}$ to $900 \mathrm{pk} / \mathrm{s}$ ) are not between the maximum and minimum values that the model provides. However, when our model uses more precise values of the parameter $T_{a}$ (i.e., ones experimentally obtained), the results are more accurate in that middle load range, as Fig. 11(b) shows.

3) Impact of the Packet Size: Besides the packet load, another parameter that strongly influences the $T_{i d l e \_} A B$ calculation is the packet transmission time. We carried out evaluations with the model by varying the mean packet size from 100 bytes to 1400 bytes for unicast data packets, which means that the transmission time of the data packets is in the range of $240 \mu \mathrm{s}$ to $1968 \mu \mathrm{s}$. In Fig. 12, $T_{i d l e \_} A B$ from our model is represented as a function of the data packet transmission time, and each curve corresponds to a different packet load case. We can see that $T_{i d l e \_} A B$ is very sensitive to the packet transmission time. The larger the packet transmission time, the lower $T_{i d l e \_} A B$. In addition, the higher the packet load, the higher the packet transmission time's impact. On the other hand, the larger the packet transmission time, the more impact the packet load has on $T_{\text {idle_AB }}$.

4) Impact of the Contention Period: In this regard, an analysis of the $T_{\text {idle } \_A B}$ sensitivity in function of $T_{c o}$ has been carried out. $T_{\text {idle_AB }}$ results from our model given in Fig. 13 show a low impact of $\widetilde{T_{c o}}$ whatever the load case. In the worst evaluated case, a deviation of approximately $50 \%$ from the real $T_{c o}$ value means a difference smaller than $5 \%$ of the real $T_{i d l e \_} A B$ value. We observe that, for cases with low packet load, $T_{i d l e \_} A B$ is almost independent of the $\widetilde{T_{c o}}$, whereas the impact increases with a load increase. That means that when the total load is low, 
for any value of $\widetilde{T_{c o}}$, we obtain a similar value for $T_{i d l e \_} A B$. In contrast, when the total load is high, an increment of $\widetilde{T_{c o}}$ means an increment of $T_{i d l e_{-} A B}$. This behaviour is consistent with the system under analysis, because if only the contention period increases, packets are retained longer and thus the medium occupancy decreases.

On the other hand, we also obtain different measures during the simulations in order to check the relevance of some assumptions in our modeling. For example, we assume that the two nodes of Link A-B maintain almost the same number of neighbors around them. We observed this property during the simulations and there were approximately 9 nodes in the transmission range and 13 nodes in the carrier sensing range. Then, the quantity of nodes in the carrier sensing area was proportional to the quantity of nodes into the transmission area (see Table III). Consequently, the assumption that nodes are uniformly distributed is a good estimation. In addition, we measured $T_{c o}$ from the simulation for each case. These values are presented in column $T_{c o}$ of Table IV and analyzed in the following section.

\section{ANALysis of the CONTENTION PERIOD USING THE FACTOR U-LOAD}

\section{A. Measurements of the Contention Period}

As we showed in Section V-C4, a very accurate value of the parameter $T_{c o}$ is not needed to evaluate $T_{i d l e_{-} A B}$. In our VANET scenarios, if the $T_{c o}$ parameter value varies, the $T_{i d l e}$ results do not vary significantly (see Fig. 13). In the worst case (case 7, the highest load), considering the slope of a linear approximation, we see that a variation of $\Delta T_{c o} \mu \mathrm{sec}$ for $T_{c o}$ implies a variation of $8 \cdot 10^{-5} \Delta T_{\text {co }} \% \mathrm{~T}$ for $T_{i d l e}$. In Section $\mathrm{V}$-B we have presented $\widetilde{T_{c o}}$, which is an approximation of $T_{c o}$ as a function of a factor that we call U-Load [see (12) and (13)].

To verify the relation among the packet rate, the packet transmission time and the contention period, we have carried out another set of simulations for a similar scenario network that we identify as Scenario 2. Note that our modeling proposal can be directly applied to different IEEE 802.11 amendments, since the MAC operation remains the same. The model just needs to set the specific parameters corresponding to the wireless technology (e.g., SIFS, DIFS, MAC header length). The parameters of Scenario 2 that differ from Scenario 1 are shown in Table VII , whereas the rest of parameters remain the same. Notice that the values of these parameters define a fictitious network rather similar to a MANET than to a VANET. We just want to use different values in the network parameters to study their influence on the behavior of a VANET.

Even though the same contention mechanism is implemented in both scenarios, there are other factors that could produce different performance. Firstly, the contention mechanism defines the backoff time in unit of time slots. Then, a proper comparison of the $T_{c o}$ values should be done in slot units. Note that the time slot is different between the two scenarios. Secondly, the backoff counter freezes while the channel is sensed busy. Then, the packet transmission time influences the period of staying in contention, besides DIFS and, in case of unicast data, SIFS
TABLE VII

SimUlation SETTINGS FOR SCENARIO 2

\begin{tabular}{lc}
\hline \hline Parameter & Scenario 2 \\
\hline MAC layer protocol & IEEE $802.11 \mathrm{~b}$ \\
MAC head length & 28 bytes \\
Ethernet II header & 14 bytes \\
PREAMBLE time & $192 \mu \mathrm{s}$ \\
SIGNAL time & - \\
SERVICE+Tail bits & - \\
Slot time & $20 \mu \mathrm{s}$ \\
SIFS & $10 \mu \mathrm{s}$ \\
DIFS & $50 \mu \mathrm{s}$ \\
Capacity channel & $3 \mathrm{Mbps}$
\end{tabular}

Only the values that differ regarding to Scenario 1 are shown.

*According to DSSS 802.11 b, preamble size of 24 bytes

transmitted at the basic rate of $1 \mathrm{Mbps}$.

** $3 \mathrm{Mb} / \mathrm{s}$ is not a possible capacity with $802.11 \mathrm{~b}$.

However, this lower fictitious capacity allows us to evaluate

a network scenario with a different $T_{i d l e}$ than in Scenario 1 ,

for the same packet rate and the same packet size.

plus ACK time. Therefore, to incorporate the influence of the packet transmission time, we define the factor U-Load as in (13). U-Load allows us to evaluate, in an easy way, the influence of several parameters which modify the $T_{u}$ value, e.g., packet headers, slot time, DIFS, SIFS...

Scenario 1 and Scenario 2 have the same quantity of nodes, the same node distribution, the same data packet sizes, and the same packet load corresponding to the different cases 1 to 7 . Nonetheless, on the opposite, they have different packet transmission times, because the channel capacity and the header times are different between the two scenarios. For example, the mean transmission time for ACK, hello and data packets is $64 \mu \mathrm{s}, 192 \mu \mathrm{s}$ and $864 \mu \mathrm{s}$, respectively, in Scenario 1, whereas in Scenario 2, the values for these times are $342 \mu \mathrm{s}, 475 \mu \mathrm{s}$ and $1820 \mu \mathrm{s}$, respectively. These different transmission times will influence differently the contention period experimented by the packets.

Fig. 14 shows the results obtained for $T_{c o}$ from simulations for both Scenario 1 and Scenario 2. In Fig. 14(a) the $T_{c o}$ values are presented in microseconds and drawn as a function of $\Lambda$ expressed in packets/second (see Table IV). We can see in Fig. 14(a) that these $T_{c o}$ values are notably higher for Scenario 2 than for Scenario 1 . This is mainly due to each packet that uses the medium longer and that therefore increases the contention period. Although the comparison of these values represents a comparative performance analysis of the two scenarios, it shows that $\Lambda$ is not the only parameter that impacts $T_{c o}$.

The $T_{c o}$ values as a function of the U-Load are depicted in Fig. 14(b). We can observe a perfect coincidence between the $T_{c o}$ values in both Scenario 1 and Scenario 2, which shows that U-Load is a good parameter to express $T_{c o}$. We can also see that all these values follow an almost straight line, thus a linear approximation seems to be appropriate.

In Fig. 14(b) this approximation and its $R^{2}$ coefficient for each scenario are drawn. The $R^{2}$ values indicate a very well fit of these regression lines. Then, we can simply approximate the $T_{c o}$ values to the linear function (12) of $\widetilde{T_{c o}}$ (in slot units). 


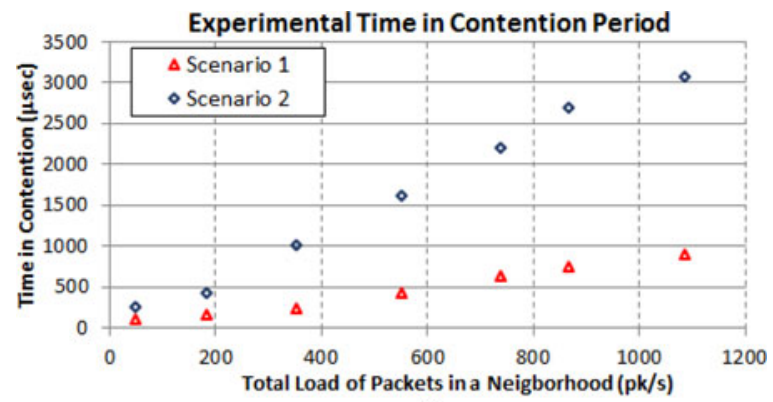

(a)

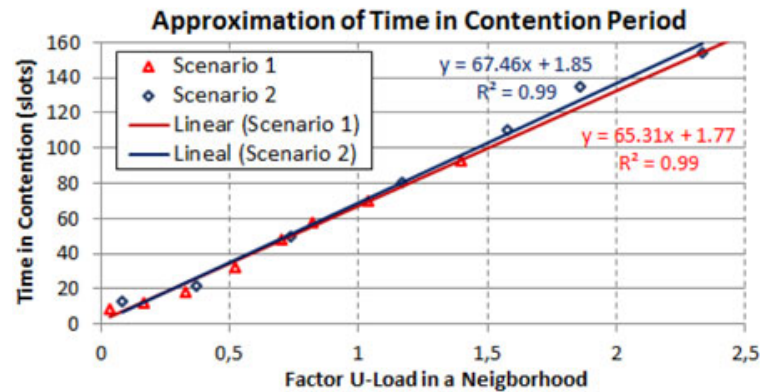

(b)

Fig. 14. Mean time that a node stays in contention $\left(T_{c o}\right)$ for each packet to be transmitted. (a) $T_{c o}$ as function of the total packet load ( $\Lambda$ ). (b) $T_{c o}$ as function of factor U-Load.

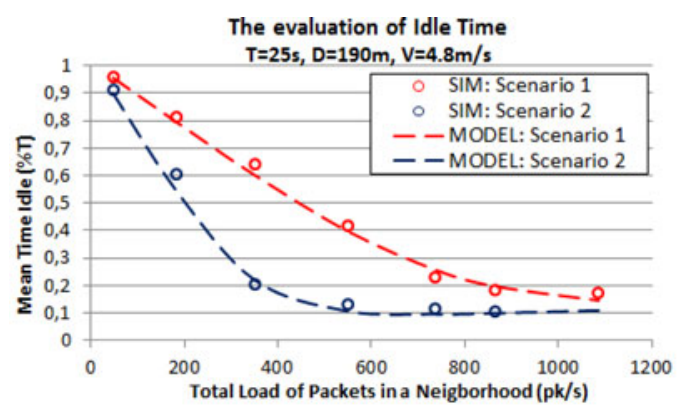

(a)

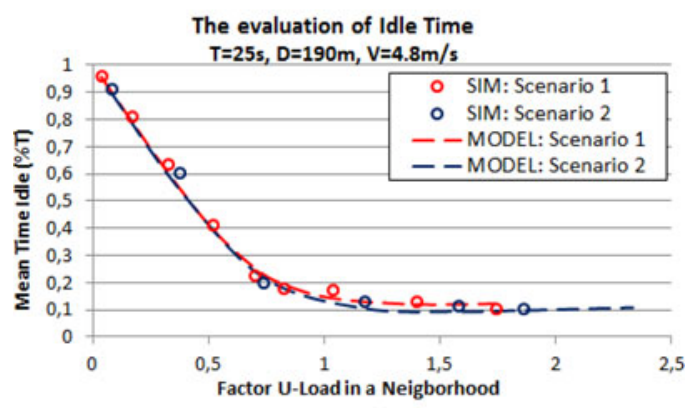

(b)

Fig. 15. Numerical results $T_{i d l e}$ in Scenario1 and Scenario2. (a) $T_{\text {idle }}$ as function of the total packet load. (b) $T_{i d l e}$ as function of factor U-Load.

\section{B. Measurements of $T_{\text {idle }}$ in a VANET as a Function of $U$-Load}

Fig. 15 shows the results for $T_{i d l e_{-} A B}$, in Scenario 1 and Scenario 2, as a function of the total packet rate $(\Lambda)$ in units of packets/second [see Fig. 15(a)] and of the factor U-Load [in Fig. 15(b)]. Due to the input parameters of Scenario 1 and
Scenario 2, for a same total packet load, the time $T_{i d l e \_A B}$ will always be less in Scenario 2 than in Scenario 1 [see Fig. 15(a)]. Nonetheless, $T_{i d l e \_} A B$ is almost the same or both Scenario 1 and Scenario 2 when they are related to their respective values of the factor U-Load [see Fig. 15(b)]. Note that the simulation results of Scenario 2 also allow us to validate that the proposed model properly characterizes the network when diverse modifications of their parameters are applied. We can observe in Fig. 15 that the modeling results fit well to the ones from simulations.

\section{CONCLUSIONS AND FUtURE WORK}

In the present work, a VANET modeling method based on Markov Rewards Chains (MRC) has been proposed. The model focuses on obtaining transient measurements of the idle time $\left(T_{i d l e}\right)$ of the communication link formed by a pair of nodes in a VANET.

The modeling parameters capture in a simple way the mobility of the nodes, the influence of the hidden nodes, the packet traffic and the packet contention. The development of an analytical model for VANETs and its evaluation has been fulfilled. We can conclude that the analytical results derived from our model for the idle time, $T_{i d l e}$, fit very well the simulation results.

Our proposal is simple enough to evaluate transient measures of $T_{i d l e}$ in links formed by VANET nodes. Additionally, our model can easily be configured to represent a dynamic VANET scenario, e.g., we could have a different VANET model throughout time along the day, for the weekend or for a working day, just varying the nodes' density.

As future work, we plan to study more deeply the relation among the packet rate, the packet transmission time and the contention period to consider heterogeneous nodes (e.g., scenarios with cross streets or with different transmission and sensing ranges). Besides, we plan to extend our proposal in an urban scenario under an emergency situation where, for instance, a crashed vehicle disseminates warning messages. In this scenario, the knowledge of the channel occupancy will surely improve the performance of the dissemination protocol. Furthermore, the presence of obstacles such as buildings will be included in our modeling. We also project to consider the impact of imperfect carrier sensing on the idle time evaluation.

\section{REFERENCES}

[1] J. Meyer, "On evaluating the performability of degradable computing systems," IEEE Trans. Comput., vol. C-29, no. 8, pp. 720-731, Aug. 1980

[2] B. R. Haverkort, R. Marie, G. Rubino, and K. S. Trivedi, Performability Modelling: Techniques and Tools. New York, NY, USA: Wiley, 2001.

[3] D. Gross and D. R. Miller, "The randomization technique as a modeling tool and solution procedure for transient Markov processes," Oper. Res., vol. 32, no. 2, pp. 343-361, Mar.-Apr. 1984.

[4] E. de Souza e Silva and H. R. Gail, "Calculating availability and performability measures of repairable computer systems using randomization," $J$. ACM, vol. 36, no. 1, pp. 171-193, Jan. 1989.

[5] J. Meyer, "Performability of an algorithm for connection admission control," IEEE Trans. Comput., vol. 50, no. 7, pp. 724-733, Jul. 2001. 
[6] I. V. Martín, J. J. Alins, M. Aguilar-Igartua, and J. Mata-Díaz, "Performability analysis of an adaptive-rate video-streaming service in end-to-end QoS scenarios," in Proc. Ambient Netw., 2005, vol. 3775, pp. 157-168.

[7] M. Garetto, T. Salonidis, and E. Knightly, "Modeling per-flow throughput and capturing starvation in CSMA multi-hop wireless networks," IEEE/ACM Trans. Netw., vol. 16, no. 4, pp. 864-877, Aug. 2008.

[8] R. Boorstyn, A. Kershenbaum, B. Maglaris, and V. Sahin, "Throughput analysis in multihop CSMA packet radio networks," IEEE Trans. Commun., vol. 35, no. 3, pp. 267-274, Mar. 1987.

[9] C. Sarr, C. Chaudet, G. Chelius, and I. Guérin-Lassous, "Bandwidth estimation for IEEE 802.11-based Ad Hoc networks," IEEE Trans. Mobile Comput., vol. 7, no. 10, pp. 1228-1241, Oct. 2008.

[10] H. Zhao, E. Garcia-Palacios, J. Wei, and Y. Xi, "Accurate available bandwidth estimation in IEEE 802.11-based ad hoc networks," Comput. Commun., vol. 32, no. 6, pp. 1050-1057, Apr. 2009.

[11] C. Tripp-Barba, M. Aguilar Igartua, L. Urquiza Aguiar, A. M. Mezher, A. Zaldívar, and I. Guérin-Lassous, "Available bandwidth estimation in GPSR for VANETs," in Proc. 3rd ACM Int. Symp. Des. Anal. Intell. Veh. Netw. Appl., Nov. 2013, pp. 1-8.

[12] R. Meireles, M. Boban, P. Steenkiste, O. Tonguz, and J. Barros, "Experimental study on the impact of vehicular obstructions in VANETs," in Proc. IEEE Veh. Netw. Conf., 2010, pp. 338-345.

[13] S. Geirhofer, L. Tong, and B. Sadler, "Cognitive medium access: Constraining interference based on experimental models," IEEE J. Sel. Areas Commun., vol. 26, no. 1, pp. 95-105, Jan. 2008.

[14] M. Lagana, I. Glaropoulos, V. Fodor, and C. Petrioli, "Modeling and estimation of partially observed WLAN activity for cognitive WSNs," in Proc. IEEE Wireless Commun. Netw. Conf., Apr. 2012, pp. 1526-1531.

[15] N. V. Nguyen, I. Guérin-Lassous, V. Moraru, and T. Razafindralambo, "Characterisation and application of idle period durations in IEEE 802.11 DCF-based multihop wireless networks," in Proc. ACM Int. Conf. Model., Anal. Simul. Wireless Mobile Syst., Oct. 2012, pp. 277-284.

[16] G. Bansal, J. B. Kenney, and C. E. Rohrs, "LIMERIC: A linear adaptive message rate algorithm for DSRC congestion control," IEEE Trans. Veh. Technol., vol. 62, no. 9, pp. 4182-4197, Nov. 2013.

[17] Y. P. Fallah, N. Nasiriani, and H. Krishnan, "Stable and fair power control in vehicle safety networks," IEEE Trans. Veh. Technol., vol. 65, no. 3, pp. 1662-1675, Mar. 2016.

[18] Y. P. Fallah, C. L. Huang, R. Sengupta, and H. Krishnan, "Analysis of information dissemination in vehicular Ad-Hoc networks with application to cooperative vehicle safety systems," IEEE Trans. Veh. Technol., vol. 60, no. 1, pp. 233-247, Jan. 2011.

[19] Q. Yang, J. Zheng, and L. Shen, "Modeling and performance analysis of periodic broadcast in vehicular ad hoc networks," in Proc. IEEE GLOBECOM, Dec. 2011, pp. 1-5.

[20] X. Ma, X. Chen, and H. H. Refai, "Unsaturated performance of IEEE 802.11 broadcast service in vehicle-to-vehicle networks," in Proc. IEEE Veh. Technol. Conf., Sep. 2007, pp. 1957-1961.

[21] X. Ma and X. Chen, "Delay and broadcast reception rates of highway safety applications in vehicular ad hoc networks," in Proc. Mobile Network. Veh. Environ., 2007, pp. 85-90.

[22] X. Ma, "On the reliability and performance of real-time one-hop broadcast MANETs," Wireless Netw., vol. 17, no. 5, pp. 1323-1337, May 2011.

[23] S. Bastani, B. Landfeldt, and L. Libman, "On the reliability of safety message broadcasting urban vehicular ad hoc networks," in Proc. 14th ACM In. Conf. Model., Anal. Simul. Wireless Mobile Syst., ACM, 2011, pp. 307-316.

[24] H. J. F. Qiu, I.-H. Ho, C. K. Tse, and Y. Xie, "A methodology for studying 802.11p VANET broadcasting performance with practical vehicle distribution," IEEE Trans. Veh. Technol., vol. 64, no. 10, pp. 4756-4769, Oct. 2015.

[25] 5G-PPP, "5G Automotive Vision," 5G-PPP, Tech. Rep., Oct. 2015.

[26] G. Bianchi, "Performance analysis of the IEEE 802.11 distributed coordination function," IEEE J. Sel. Areas Commun., vol. 18, no. 3, pp. 535-547, Mar. 2000.

[27] A. Avizienis, J.-C. Laprie, and R. B. , "Fundamental concepts of dependability," in Proc. 3rd Inf. Survivability Workshop, 2000, pp. 7-12.

[28] R. Vallejos and M. Barria, "Evaluation of moments of cumulative reward in repairable systems," Universidad Técnica Federico Santa María, Valparaso, Chile, Tech. Rep., 2005.

[29] S. Y. Wang et al., "The design and implementation of the NCTUns 1.0 network simulator," Comput. Netw., vol. 42, no. 2, pp. 175-197, Jun. 2003.

[30] L. Urquiza-Aguiar, "VANET simulations over NCTUns simulator," 2017. [Online]. Available: http://www.lfurquiza.com/research/NCTUns

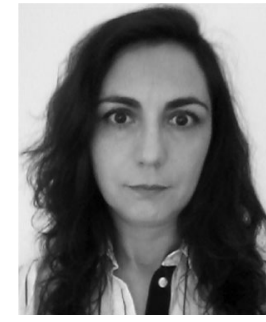

Isabel V. Martin-Faus received the M.S. degree in electronics engineering from the Universidad Técnica Federico Santa María, Valparaso, Chil, in 1999, and the Ph.D. degree from the Universitat Politècnica de Catalunya (UPC), Barcelona, Spain, in 2008. She is currently an Assistant Professor and a member of the Information Security Group with the Department of Network Engineering, UPC. Her research interests include modeling and performance evaluation for adaptive multimedia services.

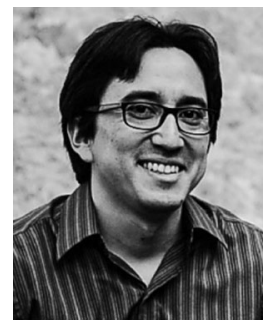

Luis Urquiza-Aguiar received the M.Sc. and Ph.D. degrees in telematics engineering from the Universitat Politècnica de Catalunya, Barcelona, Spain, in 2012 and 2016, respectively. He is a Lecturer with Escuela Politécnica Nacional, Quito, Ecuador. His research interests include span routing protocols for delay tolerant applications, wireless networks, mathematical modeling, and optimization of large-scale telecommunication problems.

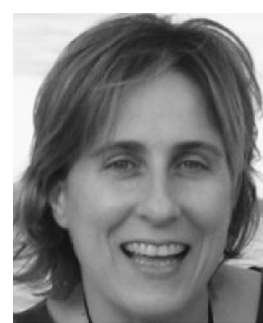

Mónica Aguilar Igartua received the M.Sc. and $\mathrm{Ph} . \mathrm{D}$. degree in telecommunication engineering from the Universitat Politècnica de Catalunya (UPC) Barcelona, Spain, in 1995 and 2000, respectively. She is an Associate Professor and a member of the Smart Services for Information Systems and Communication Networks Group with the Department of Network Engineering, UPC. Her research interests include design, analytical modelling, and performance evaluation of multimedia services over vehicular ad hoc networks (VANETs) in smart cities.

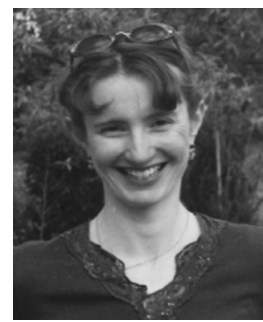

Isabelle Guérin-Lassous received the Ph.D. degree in computer science from the University Paris Diderot, Paris, France, in 1999. She has been a Professor with the Université Claude Bernard Lyon 1, France and LIP (ENSL, CNRS, UCBL, INRIA), Lyon, France, since September 2006. Her research interests include wireless networks, networks, and distributed algorithms. 